THE UNIVERSITY OF CHICAGO

\title{
DYNAMICS OF MACROECONOMIC FORECASTING VARIATION AND CORRELATION STRUCTURE IN THE BUSINESS CYCLE
}

\author{
A DISSERTATION SUBMITTED TO \\ THE FACULTY OF THE DIVISION OF THE SOCIAL SCIENCES \\ IN CANDIDACY FOR THE DEGREE OF \\ DOCTOR OF PHILOSOPHY
}

KENNETH C. GRIFFIN DEPARTMENT OF ECONOMICS

\author{
BY \\ LLOYD SANGWOO HAN
}

CHICAGO, ILLINOIS

JUNE 2019 


\section{TABLE OF CONTENTS}

LIST OF FIGURES . . . . . . . . . . . . . . . . . . . iv

LIST OF TABLES . . . . . . . . . . . . . . . . . . . . v v

ACKNOWLEDGMENTS ................................... vii

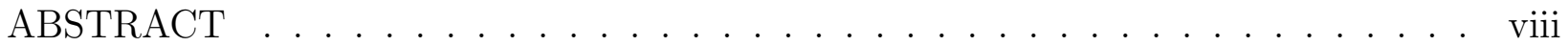

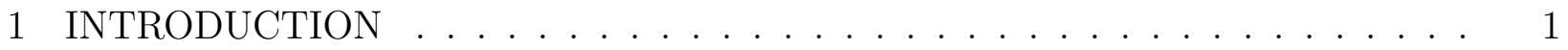

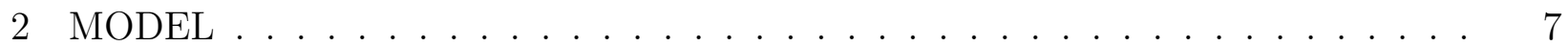

2.1 RBC Components . . . . . . . . . . . . . . . . . . . 7

2.2 Incomplete Information . . . . . . . . . . . . . . . . . . . . . . . . . . . . . . . . . . .

2.2.1 Timing Structure . . . . . . . . . . . . . . . . . . . . 9 9

2.2 .2 Signal Structure . . . . . . . . . . . . . . . . . . . 10

2.2.3 Uncertainty and Disagreement . . . . . . . . . . . . . . . 14

2.3 Fears of Model Misspecification . . . . . . . . . . . . . . . . . 16

2.4 Equilibrium and Value Function . . . . . . . . . . . . . . . . . . . . 18

2.4.1 Recursive Equilibrium . . . . . . . . . . . . . . 18

3 SOLUTION METHOD . . . . . . . . . . . . . . . . . . . . . . . . . . . . . . . . . . . . . .

3.1 Data Generating Process . . . . . . . . . . . . . . . . . 21

3.2 Optimal Controls . . . . . . . . . . . . . . . . . . . . . . . . . . . . . . . . . . . . . . .

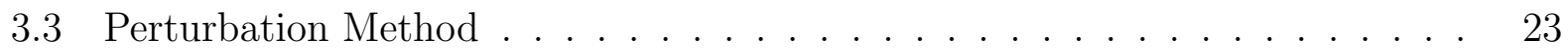

4 LINKING UNCERTAINTY AND DISAGREEMENT TO DATA . . . . . . . . . 29

5 SURVEY EVIDENCE . . . . . . . . . . . . . . . . . . . . . . . 31

5.1 Constructing Probability Densities . . . . . . . . . . . . . . . . 33

5.2 Summary Statistics of Forecasts . . . . . . . . . . . . . . . . . . . . . . . . . . . . . . . . . 33

5.3 Constructing Uncertainty and Disagreement . . . . . . . . . . . . . . 35

6 CALIBRATION . . . . . . . . . . . . . . . . . . . . . . . . . . . 38

6.1 Macro Parameters . . . . . . . . . . . . . . . . . . . . 38

6.2 Uncertainty and Disagreement . . . . . . . . . . . . . . . . . . . . . . . . . . . . . . . . . . .

6.3 Fears of Model Misspecification . . . . . . . . . . . . . . . . . . . 39

7 RESULTS . . . . . . . . . . . . . . . . . . . . . . . . . . . 42

7.1 Uncertainty and Disagreement on Aggregate Variables . . . . . . . . . . . . . 42

7.2 Impulse Response Functions . . . . . . . . . . . . . . . . . . . . . . . . . . 44

7.3 Distribution of Signals . . . . . . . . . . . . . . . . . . . . . 45

7.4 Magnitude of Probability Distortions . . . . . . . . . . . . . . . . 46

7.5 Aggregate Moments . . . . . . . . . . . . . . . . . . . . 47 


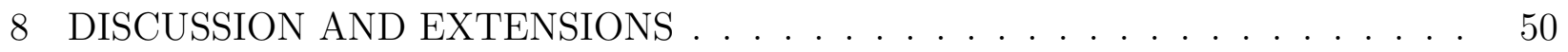

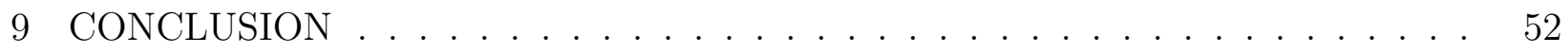

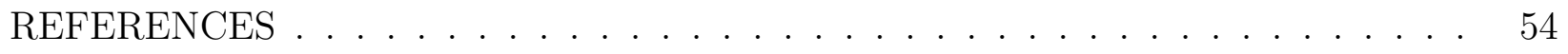

A APPENDIX: US AGGREGATE DATA SOURCES . . . . . . . . . . . . 56

B APPENDIX: EXTRACTING UNCERTAINTY AND DISAGREEMENT . . . . . 57

B.1 Time Period and Distributional Assumptions Within Bins . . . . . . . 57

B.2 Evidence against the Effect of Binning . . . . . . . . . . . . 61

B.3 Robustness of Properties of Uncertainty and Disagreement . . . . . . . . . 63

B.4 Role of Recent Financial Crisis . . . . . . . . . . . . . . . . . . . . 65

C APPENDIX: LINKING REAL GDP GROWTH IN SURVEY DATA TO PRODUCTIVITY IN THE MODEL . . . . . . . . . . . . . . . . . . . . 67

C.1 Forecasting Real GDP Growth with Aggregate Labor Change . . . . . . . . 67

C.2 Comparing Real GDP Growth and Productivity . . . . . . . . . . . . 70 


\section{LIST OF FIGURES}

5.1 Plot of the moments of the densities extracted from SPF survey responses when asked to predict a probability distribution for the real GDP growth rate. $\hat{\psi}_{\left.t\right|_{o}}$ represents the average forecasted growth rate, $\hat{\chi}_{t \mid o}$ represents dispersion within a forecasters forecast, and $\hat{\xi}_{t \mid o}$ represents dispersion across forecasters. Plot generated using $o=4$. Red highlights shows NBER recession periods. The plotted date is the date when the forecast is made. All values are annualized and in percent. 37

7.1 Model predicted response of aggregate variables to a one time one standard deviation shock to uncertainty and disagreement. Uncertainty shown in the left panel and disagreement shown in the right panel. All variables shown as percent deviations from steady state. . . . . . . . . . . . . . . . .

7.2 Implied time series for the signal volatility $\left(w_{t}\right)$ and correlation $\left(\tau_{t}\right)$ using uncertainty and disagreement fit to SPF data setting $o=4$. Red highlights show NBER recession periods. Plotted date is when forecast is made. Values are for



B.1 Plot comparing PDFs for the uniform, normal, and beta distributional assumption for examples of responses in the SPF data. Blue shows the uniform fit results, red shows the normal fit results, and yellow shows the beta fit results. All values are annualized and in percent. . . . . . . . . . . . . .

B.2 Plot comparing estimates of $\hat{\psi}_{t \mid o}, \hat{\chi}_{t \mid o}$, and $\hat{\xi}_{t \mid o}$ using the uniform, normal, and generalized beta distribution fit for $o=4$. Blue shows the uniform fit results, red shows the normal fit results, and yellow shows the beta fit results. Red highlights show NBER recession periods. All values are annualized and in percent. . . . . .

B.3 Plot comparing estimates of $\hat{\psi}_{t \mid o}, \hat{\chi}_{t \mid o}$, and $\hat{\xi}_{t \mid o}$ using a uniform, normal, and generalized beta distribution with $o=4$ for ECB data. Blue shows the uniform fit results, red shows the normal fit results, and yellow shows the beta fit results. Red highlights show NBER recession periods. All values are annualized and in percent. . . . . . . . . . . . . . . . . .

B.4 Plot of estimates of uncertainty from the MSC and disagreement from IBES. Red highlights show NBER recession periods. All values are annualized and in percent. 64

C.1 Plot of fitted $\chi_{t}, \xi_{t}$ minimizing the objective in Equation (C.9) and the estimators $\hat{\chi}_{t \mid o}, \hat{\xi}_{t \mid o}$. I plot the estimators in blue, and the fitted values in dotted red. Red highlights indicate NBER recession periods. All variables annualized and in percent. 69 


\section{LIST OF TABLES}

5.1 Summary statistics for the forecasts, forecast errors, and forecast revisions for both the density and point estimates for real GDP. Forecast revisions are computed using the forecast in the previous quarter. The SD for the density forecast is the standard deviation across forecasters of the fitted mean of the density estimate. All values annualized and in percent. . . . . . . . . . . . . .

6.1 Baseline parameter values. Model is calibrated for annual data. The evolution of

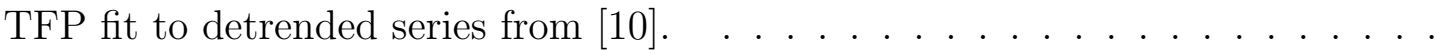

6.2 Uncertainty and disagreement parameter values. Values fit to data from SPF

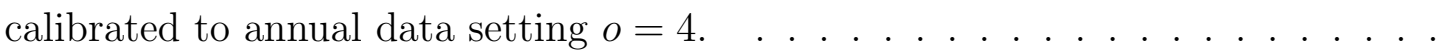

6.3 Effects of uncertainty and disagreement on US aggregate variables. Aggregate variables represented as percent deviations from steady state. Productivity, uncertainty, and disagreement represented in percent. Newey-West robust standard errors shown. . . . . . . . . . . . . . . . . . . . .

6.4 Data and model predicted effects of uncertainty and disagreement on aggregate variables. $\theta$ is calibrated to fit an evenly weighted sum of the effect of uncertainty and disagreement on labor and consumption. Aggregate variables represented as percent deviations from steady state. Productivity, uncertainty, and disagreement represented in percent. Newey-West standard errors from the regression shown in parenthesis. . . . . . . . . . . . . . . . . . . .

7.1 Data and model simulated aggregate moments. Moments represented as percent deviation from steady state. US aggregate data shown in the left column. Results from three experiments and comparison with results from [1] shown. . . . . . . .

A.1 Data Sources for US Macrodata. . . . . . . . . . . . . . . .

B.1 Comparison of coefficients from the regression in equation (6.1) for varying distributional assumptions. Aggregate variables represented as percent deviations from steady state. Productivity, uncertainty, and disagreement represented in percent. Newey-West robust standard errors shown. . . . . . . . . . . . . . .

B.2 Data and model predicted effects of uncertainty and disagreement on aggregate variables for normal and beta distributional fits. $\theta$ is calibrated to fit an evenly weighted sum of the effect of uncertainty and disagreement on labor and consumption. Aggregate variables represented as percent deviations from steady state. Productivity, uncertainty, and disagreement represented in percent. Newey-West standard errors from the regression shown in parenthesis. . . . . . . . . . . .

B.3 Effects of uncertainty and disagreement on US aggregate variables excluding year 2009. Uniform distributional assumption used. Aggregate variables represented as percent deviations from steady state. Productivity, uncertainty, and disagreement represented in percent. Newey-West robust standard errors shown. . . . . 
C.1 Contemporary regression of TFP on real Output. All variables represented as percent deviation from steady state. Results for the full sample 1960-2017 and the sample consistent with SPF data 1992-2017 shown. Spherical standard errors shown. 


\section{ACKNOWLEDGMENTS}

I would like to thank my advisors Lars P. Hansen (co-chair), Harald Uhlig (co-chair), Yueran Ma, and Mikhail Golosov for their advice and support. I would also like to thank participants of the Economic Dynamics and Financial Markets working group at the University of Chicago for helpful comments. 


\begin{abstract}
Using a structural model, I analyze how changes in the distribution of signals about unknown economic conditions affect real aggregate macrovariables in the business cycle. I focus on two quantifiable properties of the distribution of signals, the signal accuracy and the correlation structure across signals, and analyze how time variation in these two properties affect an agent's decisions through his posterior beliefs. Since the exact signals agents use are difficult to observe empirically, I define two key concepts, uncertainty and disagreement, that capture dynamics in the distribution of signals and can be linked to data. Uncertainty is defined as the dispersion in each agent's forecasts about economic conditions. Disagreement is defined as the dispersion across agents in their mean forecasts about economic conditions. I show that uncertainty and disagreement affect an agent's controls through his first and higher order beliefs about economic conditions. Calibrating to US macrodata and the Survey of Professional Forecasters, I show empirically that the distribution of signals matters for aggregate dynamics and that my model mechanism can parsimoniously match the magnitude and sign of these effects. However, I find movements in the distribution of signals represent only a small fraction of the total variation in aggregate variables.
\end{abstract}




\section{CHAPTER 1 INTRODUCTION}

Over the course of the business cycle, economic agents make decisions such as choosing how many workers to hire or how much to invest and produce without knowing aggregate economic conditions. To formulate expectations about the unknown, agents can potentially use a wide variety of available sources. For example, analyst forecasts, news, or the Federal Open Market Committee minutes can all be informative about the future state of the economy. Imagine each agent processes these sources into a private signal about economic conditions. Naturally, properties of the available information should affect the resulting distribution of signals that agents extract. For instance, analyst forecasts can be clearly-written and precise or vague and unclear. Different analysts can report similar predictions or have very different opinions.

In this paper, I use a structural model to analyze how changes in the distribution of signals about unknown economic conditions affect real aggregate variables in the business cycle. I focus on two quantifiable properties of the distribution of signals, the signal accuracy and the correlation structure across signals, and analyze how time variation in these two properties affect an agent's choice of controls through his posterior beliefs.

I interpret dynamics in the signal accuracy and the correlation structure as capturing variation in the quality and dispersion of information sources. Going back to my example, if analyst forecasts are vague and unclear, I interpret the signal agents extract from this source of information to have lower signal accuracy. If different analysts all report similar predictions, I interpret the signal agents extract as having higher correlation across agents. In this sense, changes in signal accuracy and correlation structure capture changes in available information on the distribution of signals available to agents.

Since it is difficult to observe the precise sources and methods agents use, I abstract away from modeling how each agent formulates his signal. Instead, I impute properties of the re- 
sulting distribution of signals using survey data in which respondents are asked to forecast macroeconomic outcomes. To that end, I define two key concepts that I refer to throughout this paper as uncertainty and disagreement. Informally, uncertainty measures volatility in an agent's own posterior belief about economic conditions, and disagreement measures volatility in an agent's posterior belief about other agent's expectations for economic conditions. I show that uncertainty and disagreement map one-to-one into signal accuracy and the correlation structure across signals, and I show how to extract a time-series for uncertainty and disagreement directly from survey data.

My model assumes there is a continuum of agents that each produce intermediate goods which are complementary. I represent unknown economic conditions by letting productivity which is common to all agents be unknown and stochastic. Each agent commits to a level of production given his private signal for productivity which may be correlated with the signal of other agents. Complementarity of produced goods implies that each agent must not only forecast the unknown productivity but also forecast the forecasts of other agents. As a result, both an agent's first and higher order beliefs about productivity play a role in the model. Intuitively, uncertainty affects first order beliefs (i.e. what I think about productivity) while disagreement affects higher order beliefs (i.e. what I think other people think about productivity). Through this belief channel, dynamics in the distribution of signals about productivity enter each agent's decision problem.

A key component in the model is the addition of robustness concerns. I endow agents with fears of potential model misspecification for two reasons. First, robustness allows me to tractably incorporate dynamics in the distribution of signals. Second, robustness allows me to quantify the empirical success of my model.

A germane problem in the literature among models with higher order beliefs has been to incorporate enough richness in the specification of beliefs while maintaining tractability. ${ }^{1}$

1. For examples of approaches in the literature see [2], [12], and [14] among others. 
Given my focus on dynamics in the distribution of signals, I use a perturbation method around the non-stochastic steady state to solve the model that is based on [15]. To a first order expansion, I show each agent's beliefs can be represented as a posterior distribution for the unknown productivity and the average signal. The dependence on both the productivity and average signal highlight the separate dependence on first and higher order beliefs. The posterior for productivity represents an agent's own expectations about productivity. The posterior for the average signal represents an agent's expectations about other agents' expectations. Time-variation in uncertainty and disagreement drive movements in the mean and variance of this posterior which affect an agent's choice of controls.

However, with a first order expansion, neither changes in the posterior mean nor variance affect the dynamics of an agent's controls. Higher order expansion terms could be used to capture these effects, however, in doing so, the posterior distribution for productivity and the average signal are no longer sufficient to capture higher order beliefs. In fact, infinitely many orders of beliefs are required capture the decision problem of each agent.

To address this problem, I endow agents with fears of model misspecification using the multiplier preferences from [11]. Each agent considers a set of distorted probability measures parameterized by a strictly positive function which acts as a Radon-Nikodym derivative. To restrict the set of possibilities, the agent penalizes the log likelihood ratio between potential alternatives and the original baseline specification. The agent then solves a max-min problem minimizing over his choice of model then maximizing over his choice of controls. The multiplier preference structure adds additional curvature to the continuation value of each agent. I find the choice of the minimizing model, which I refer to as the worst case model, is a function of the posterior variance causing movements in the signal distribution to affect an agent's controls through beliefs while keeping a tractable structure.

In addition, the multiplier preferences introduce an additional free parameter: the Lagrange multiplier on the log-likelihood ratio constraint. I show that the multiplier scales the 
magnitude of the impact of uncertainty and disagreement on the agent's controls. By computing the worst case model associated with the value for the multiplier in my calibration, I evaluate, using statistical discrepancy measures, the empirical plausibility of my model mechanism and results.

To extract time-series for uncertainty and disagreement, I use data from the Survey of Professional Forecasters (SPF) on forecasts of the growth rate of real GDP. In addition to point forecasts, the SPF asks for density forecasts: respondents are asked to assign a probability that the growth rate lies in a number of discrete bins. The data gives me a discrete estimate of the cumulative distribution function for the growth rate for each respondent at each point in time. I approximate a probability density function from the discrete bins using assumptions on the structure of the underlying distribution. This process then allows me to compute moments of each respondent's distribution for the growth rate at each point in time.

To connect the SPF data to my model, I take the stance that survey respondents have the same probability views as the agents in my model. In addition, I assume respondents provide the objective probability measures to the survey, and agents incorporate the cautious adjustment for potential model misspecification in their choice of controls. By comparing forecasts to the true values, I find survey respondents on average predict the growth rate accurately. The lack of systematic under prediction supports the view that respondents provide an undistorted probability in their responses as opposed to a worst case model distorted by robustness concerns.

Given these assumptions, I show that uncertainty at a point in time can be extracted taking the average across respondents of the volatility in each respondent's distribution, while disagreement can be extracted taking the volatility across respondents of the mean in each respondent's distribution. My definition of uncertainty differs from the construction of uncertainty measures in the existing literature. Often, the dispersion across forecasters is 
used as a measure for forecaster uncertainty about future outcomes. However, this dispersion is measured using point forecasts which do not contain any measure of how unsure each individual forecaster is about their reported value. As a result, this measure confounds variation in beliefs across forecasters as well as the manner in which forecasters transform beliefs into point predictions. I distinguish uncertainty and disagreement using variation within and across forecasters respectively.

To quantify the success of my model mechanism, I first show in reduced form that my extracted time-series for uncertainty and disagreement have statistically significant impact on US aggregate variables controlling for variation in productivity. I find an increase in uncertainty and disagreement both cause hours worked, consumption, investment, and output to fall. In addition, I find that the magnitude of the effect of uncertainty is larger than the effect of disagreement.

I then fit the stochastic process for uncertainty and disagreement in my model to the extracted time-series. Calibrating the other model parameters to standard values from the literature, I then show that my model mechanism can parsimoniously capture the sign and magnitude of the effects of uncertainty and disagreement consistent with the reduced form results. In addition, I verify that the degree of fears of model misspecification required to match the data results in a worst cast model which is statistically close to the original baseline model.

My paper relates to literature exploring the role of higher order beliefs in the macroeconomy. My model utilizes elements from [1]. In their paper, they show higher order beliefs can achieve a remarkably good fit of aggregate moments. In contrast to their work, my model introduces deviations in both first and higher order beliefs endogenously. I focus on the effects coming from changes in the distribution of signals and use uncertainty and disagreement to calibrate movements in beliefs directly rather than fitting a process indirectly through aggregate moments. 
Under my calibration, I find that movements in uncertainty and disagreement have no meaningful impact on aggregate moments and cannot explain the fit of moments achieved in their paper. I find my model mechanism, while having a statistically significant impact on agent's choices, cannot be the main source of the movements in higher order beliefs used in their paper and do not represent a large fraction of variation in aggregate variables.

My paper is also closely related to [3]. Using the Michigan Survey of Consumers, they find survey respondents have an counter-cyclical upward bias in predictions of unemployment and inflation. Since robustness causes agents to consider a distorted worst case model, they attribute the bias and its dynamics to changing concerns for model misspecification and analyze its effects on unemployment and inflation dynamics.

In contrast to their work, I find no systematic bias in predictions in the SPF data. ${ }^{2}$ Given this finding, I assume that agents provide an undistorted prediction to the SPF and incorporate fears of model misspecification in their choice of controls. In addition, I do not consider time-variation in concerns for robustness and focus on dynamics in the signal distribution. However, I rely on and extend their perturbation method in order to solve my model.

The rest of the paper is structured as follows. I construct the model in Chapter 2 . I explain the perturbation method used to solve the model in Chapter 3. I show how I link uncertainty and disagreement to measurable quantities in survey data in Chapter 4. I analyze the SPF data and extract time-series for uncertainty and disagreement in Chapter 5. I describe the model calibration process in Chapter 6. I highlight the results in Chapter 7. I discuss potential extensions in Chapter 8, and Chapter 9 concludes. [4].

2. I compute bias using forecast errors following the method of [7]. My findings are also consistent with 


\section{CHAPTER 2 \\ MODEL}

My model consists of a standard real business cycle (RBC) model with two additional ingredients: incomplete information and fears of model misspecification. I breakup the introduction of my model into three parts. First, I introduce the RBC components which make up the production and labor in the economy. These components allow me to consider the real aggregate consequences for output, consumption, labor and investment. I make these components "off-the-shelf" to focus on the effects from the distribution of signals and agent's expectations.

Second, I discuss the signal structure and incomplete information. I incorporate dynamics in the distribution of signals by letting the signal accuracy and the correlation of signals across agents evolve stochastically over time. I then derive how changes in the distribution of signals affect each agent's first and higher order beliefs. Given that it is hard to empirically observe the signals agents use to formulate expectations, I define two moments of the distribution of signals, uncertainty and disagreement, which capture the relevant movements in the distribution of signals and can be linked to survey data.

Third, I introduce fears of model misspecification. I show model ambiguity causes each agent to distort his first and higher order beliefs away from a baseline specification. I find un-

certainty and disagreement affect each agent's choice of labor and consumption through these distortions. After introducing the model, I define a recursive equilibrium that determines a solution to my model.

\section{$2.1 \quad$ RBC Components}

Time is discrete indexed by $t \in\{0,1, \ldots\}$. There is a continuum of agents indexed by $i \in[0,1]$. Each agent is endowed with labor and capital that produces an intermediate good 
and has time separable utility with time discount rate $\beta$

$$
\max _{\left\{c_{i, t}, n_{i, t}\right\}} \sum_{t=0}^{\infty} \beta^{t}\left\{-\frac{n_{i, t}^{1+\nu}}{1+\nu}+\frac{c_{i, t}^{1-\gamma}}{1-\gamma}\right\}
$$

where $c_{i, t}, n_{i, t}$ are the agent's choice of consumption and labor. $\gamma$ is the risk aversion and $\nu$ is the inverse of the Frisch elasticity of labor supply. Each agent is endowed with production technology for an intermediate good

$$
y_{i, t}=\exp \left(s_{t}\right) k_{i, t}^{\alpha} n_{i, t}^{1-\alpha}
$$

where $\exp \left(s_{t}\right)$ is the stochastic aggregate productivity that is common to all agents, and $k_{i, t}$ is the his own capital stock. The resource constraint for agent $i$ is given by

$$
c_{i, t}+k_{i, t+1}=p_{i, t} y_{i, t}+(1-\delta) k_{i, t}
$$

where $p_{i, t}$ is the price of the intermediate good and $\delta$ is depreciation.

Each agent trades intermediate goods for final goods which are used for consumption and investment in a competitive market. The final good is produced using a Cobb-Douglas aggregate of intermediate goods. Final goods production follows

$$
\begin{aligned}
\log Y_{t} & =\int_{0}^{1} \log y_{i, t} d i \\
\frac{p_{i, t}}{P_{t}} & =\frac{Y_{t}}{y_{i, t}}
\end{aligned}
$$

where $Y_{t}$ is the quantity of final good. Without loss of generality, I normalize $P_{t}=1$ which makes the final good the numeraire. 
I assume log productivity $s_{t}$, common to all agents, has evolution given by

$$
s_{t+1}=\rho_{s} s_{t}+\sigma_{s} \epsilon_{t+1}^{s}
$$

where $\rho_{s}, \sigma_{s}$ are parameters and $\epsilon_{t+1}^{s}$ is standard normal independent for all $t$. I note that I fix $\sigma_{s}$ to be constant time. While the changes in the distribution of signals introduced in the following sections introduce stochastic volatility, I do not allow for this variation in the productivity. I discuss the relevance of this assumption when I calibrate the process for productivity in Chapter 6.

The RBC components allow both first and higher order beliefs to enter each agent's decision. First order beliefs enter in an agent's expectations about productivity st. The aggregation of intermediate goods into a final good creates complementarity across agents in the economy. If all other agents produce more, each agent's marginal product increases. To make an optimal choice of production, each agent then has to forecast the production of other agents allowing higher order beliefs to potentially play a role through the expected intermediate goods price $p_{i, t}$.

\subsection{Incomplete Information}

\subsubsection{Timing Structure}

I introduce incomplete information into the economy by assuming that the productivity of intermediate goods production $s_{t}$ is not known. Each agent instead receives a private signal about productivity which they use to formulate expectations. Since both your own expectations as well as your expectations about the expectations of other agents matter, I use the timing convention from [1] to avoid the "infinite regress problem." ${ }^{1}$ I split each time

1. The "infinite regress problem" from [16] refers to the problem where infinitely many state variables are required to capture each agent's posterior for the beliefs of the other agents in the economy. 
period into two stages.

- Stage 1: $s_{t}$ is drawn but not known. Each agent receives a private signal about productivity and chooses labor with incomplete information about the labor choices of other agents.

- Stage 2: $s_{t}$ is revealed along with the labor choices of all agents, and production occurs. The competitive markets for intermediate and final goods acts. Each agent chooses consumption and investment.

The timing convention allows me to segment the information structure such that in each time period, an agent does not need the whole history of production of other agents to formulate expectations. This simplifying assumption has consequences for the implications of the model. Namely, I do not allow agents to learn. Since the unknown productivity is revealed in stage 2 of each time period, I assume agents do not adjust their first and higher order beliefs given the new information. I discuss these implications in more detail in Chapter 3.

\subsubsection{Signal Structure}

In Stage 1 of time $t$, agent $i$ receives a private signal $z_{i, t}$ which is distributed as

$$
\begin{gathered}
z_{i, t}=s_{t}+w_{t} \epsilon_{i, t}^{\hat{z}} \\
\operatorname{Corr}\left(\epsilon_{i, t}^{\hat{z}}, \epsilon_{j, t}^{\hat{z}}\right)=\tau_{t} \text { for } i \neq j
\end{gathered}
$$

where $\epsilon_{i, t}^{\hat{z}}$ is a standard normal shock independent of $\epsilon_{t}^{s}$ for all $i, t$. $w_{t}, \tau_{t}$ are exogenous stochastic variables common to all agents. $w_{t}$ represents the inverse of the precision of the signal, and $\tau_{t}$ the correlation of signal errors across agents. I assume that $w_{t}, \tau_{t}$ are publicly known in stage 1 at time $t$. 
I allow the accuracy of the signal and the correlation of the signal across agents to be stochastic to model changes in the distribution of signals agents receive. For example, agents in the economy go out and gather sources of information and process this raw data into a signal about future productivity. I interpret the private signal in my model as representing this processed signal. My model captures properties of the private signal of each agent resulting from qualitative properties of the information sources.

If analyst forecasts are vague and unclear or do not provide a clear convincing argument, I interpret that the private signal extracted by agents from this source of information would have lower signal accuracy (higher $w_{t}$ ). Since the raw information is less informative, I interpret this as causing the private signals of each agent to be less informative about productivity. If multiple analysts provide similar forecasts, I interpret this as corresponding to higher correlation in the signals across agents. Since the available raw information has similar content, I model the extracted private signal of each agent as having higher correlation $\tau_{t}$ across agents.

My assumption that $w_{t}, \tau_{t}$ are common to all agents is a simplifying assumption for tractability. In my example, I assumed these agents all used publicly available sources of information which is consistent with a common $w_{t}, \tau_{t}$. This assumption rules out subscribing to private data sets or analytic tools that not all agents in the economy can access. In my model $w_{t}, \tau_{t}$ are stochastic and are state variables for each agent's decision problem. To bound the state space, I assume they do not vary across agents.

I restrict $w_{t} \in[0, \infty)$ as a sign convention. In my model the agent's decision and my definition of uncertainty and disagreement will depend on $w_{t}^{2}$. To identify $w_{t}$, I assume its value is positive. In addition since $w_{t}$ will be stochastic, I do not allow $w_{t}$ to be negative to avoid a potential kink in the evolution of $w_{t}^{2}$ when $w_{t}$ crosses zero.

I restrict $\tau_{t} \in[0,1]$ to focus on cases where agents tend to be optimistic $\left(z_{i, t}>s_{t}\right)$ or pessimistic $\left(z_{i, t}<s_{t}\right)$ together. This herd mentality is consistent with psychological evidence 
as well as strategic complementarity explored in previous literature using higher order beliefs ([2]). In addition, the restriction on $\tau_{t}$ allows me to interpret the error in my signal as being composed of a systemic and idiosyncratic component. The same correlation structure of signals can be achieved via the decomposition

$$
\epsilon_{i, t}^{\hat{z}}=w_{t} \sqrt{\tau_{t}} \epsilon_{t}^{\hat{u}}+w_{t} \sqrt{1-\tau_{t}} \epsilon_{i, t}^{\hat{v}}
$$

where $\epsilon_{t}^{\hat{v}}$ represents a standard normal shock common to all agents, and $\epsilon_{i, t}^{\hat{u}}$ represents agent specific noise.

Given the signal structure, the expected sample variance of the distribution of signals across agents can be computed as

$$
\lim _{N \rightarrow \infty} \mathbb{E}_{t}\left\{\frac{1}{N-1} \sum_{j=1}^{N}\left[z_{j, t}-\frac{1}{N} \sum_{k=1}^{N} z_{k, t}\right]^{2}\right\}=w_{t}^{2}\left(1-\tau_{t}\right)
$$

Note that as $w_{t}$ increases, both the variance of the individual signal of each agent as well as the dispersion of the signals across agents increases. As $\tau_{t} \rightarrow 1$, the signals across agents become more correlated decreasing the dispersion of signals while leaving the individual signal variance unchanged.

Due to complementarity in goods, agents must formulate first and higher order beliefs about productivity in making their labor decision. I assume and later verify using a perturbation method in Chapter 3 that to a first order approximation, each agent's decision problem depends only on first and second order beliefs about productivity summarized using a posterior distribution for $s_{t}$ (first order belief) and the average signal of other agents $\zeta_{t} \equiv \int_{0}^{1} z_{j, t} d j$ (second order belief). I refer to $\zeta_{t}$ as second order beliefs since the posterior $\zeta_{t} \mid z_{i, t}$ represents the beliefs of agent $i$ about the signals of other agents in the economy. In the model each agent uses his signal to predict productivity, thus the posterior for $\zeta_{t}$ represents 
what I think other people think about productivity.

The posterior distribution for each agent is then given by ${ }^{2}$

$$
\begin{gathered}
{\left[\begin{array}{c}
s_{t} \\
\zeta_{t}
\end{array}\right] \mid z_{i, t} \sim \mathcal{N}\left(\left[\begin{array}{c}
\rho_{s} s_{t-1}+\frac{\sigma_{s}^{2}}{\sigma_{s}^{2}+w_{t}^{2}}\left(z_{i, t}-\rho_{s} s_{t-1}\right) \\
\rho_{s} s_{t-1}+\frac{\sigma_{s}^{2}+\tau_{t} w_{t}^{2}}{\sigma_{s}^{2}+w_{t}^{2}}\left(z_{i, t}-\rho_{s} s_{t-1}\right)
\end{array}\right], \tilde{\Sigma}_{t}^{s, \zeta}\right)} \\
\tilde{\Sigma}_{t}^{s, \zeta} \equiv\left[\begin{array}{cc}
\frac{w_{t}^{2}}{\sigma_{s}^{2}+w_{t}^{2}} \sigma_{s}^{2} & \frac{w_{t}^{2}\left(1-\tau_{t}\right)}{\sigma_{s}^{2}+w_{t}^{2}} \sigma_{s}^{2} \\
\frac{w_{t}^{2}\left(1-\tau_{t}\right)}{\sigma_{s}^{2}+w_{t}^{2}} \sigma_{s}^{2} & \frac{w_{t}^{2}}{\sigma_{s}^{2}+w_{t}^{2}}\left(1-\tau_{t}\right)\left(\sigma_{s}^{2}+\tau_{t} w_{t}^{2}\right)
\end{array}\right]
\end{gathered}
$$

Note that movements in the signal accuracy and correlation $w_{t}, \tau_{t}$ cause movements in both the mean and variance of the posterior distribution. Looking at the mean, I find changes in the distribution of signals affect the sensitivity of the posterior mean to the surprise in their signal, measured using the difference of the signal from its the expected value. When signal accuracy is lower $\left(w_{t}\right.$ is higher), the posterior mean of $s_{t}$ and $\zeta_{t}$ responds less to surprises in the signal. In the corner case where $\tau_{t}=1$, the effect of $w_{t}$ on the posterior mean of $\zeta_{t}$ is zero. When the signals are more correlated across agents $\left(\tau_{t}\right.$ is higher), I note that the mean of $s_{t}$ is not affected but the mean of $\zeta_{t}$ becomes more sensitive to signal surprises.

Looking at the covariance matrix, I find the effects of the changing signal distribution have a clear sign for first order beliefs. A lower signal accuracy causes a higher posterior variance of $s_{t}$, and changing the correlation across signals has no effect. For second order beliefs, an increase in $w_{t}$ increases the posterior variance of $\zeta_{t}$. The effect of increasing $\tau_{t}$ is unclear. When $\tau_{t}$ increases, on the one hand everyone's signals are more correlated decreasing the variance of $\zeta_{t}$, on the other hand, the variance becomes more sensitive to the signal accuracy $w_{t}$.

2. To compute the variance and covariance of $\int_{0}^{1} \epsilon_{j, t}^{\hat{z}} d j$, I take the limit after discretizing the interval $[0,1]$ into $N$ pieces 


\subsubsection{Uncertainty and Disagreement}

In the previous section, I derived how first and second order beliefs are affected by changes in the distribution of signals. However, it is difficult to observe empirically which sources of information agents use and how agents process this information into a signal, making calibrating processes for $w_{t}, \tau_{t}$ a challenge. To address this problem, I define two moments of the distribution of signals, uncertainty and disagreement, that I show have empirical counterparts in Chapter 4 . Let $a_{t}, b_{t}$ denote uncertainty and disagreement respectively about $s_{t}$. I define $a_{t}$ as posterior standard deviation for productivity $\left(s_{t}\right)$. I define $b_{t}$ as the standard deviation across agents of each agent's posterior mean for $s_{t}$. The posterior mean being a linear function of the private signal $z_{i, t}$, I scale the linear coefficient by the standard deviation of the signal across agents to find $b_{t}$. Given the preceding, I define uncertainty and disagreement as

$$
\begin{aligned}
a_{t} & \equiv \sigma_{s} \sqrt{\frac{w_{t}^{2}}{\sigma_{s}^{2}+w_{t}^{2}}} \\
b_{t} & \equiv \frac{\sigma_{s}^{2}}{\sigma_{s}^{2}+w_{t}^{2}} w_{t} \sqrt{1-\tau_{t}}
\end{aligned}
$$

Intuitively, uncertainty measures how unsure each agent is about his own expectations for productivity. It represents the dispersion within each agent's subjective belief about productivity. Disagreement measures the dispersion across agents in their beliefs about productivity. It measures lack of consensus among agents in their mean forecast for productivity. As a result, it represents a measure of how unsure each agent is about what other agents predict as productivity. Alternatively, one can think of uncertainty and disagreement as representing components of a variance decomposition. Uncertainty represents the within variance, where as disagreement represents the between variance.

I note that $a_{t}, b_{t}$ capture the variation in the distribution of signals parameterized by movements in $w_{t}, \tau_{t}$. Note that changes in signal accuracy lead to changes in both uncertainty 
and disagreement. For uncertainty, the change is monotonic: as $w_{t}$ increases, $a_{t}$ increases leading to higher uncertainty. For disagreement, the sign of the effect depends on two opposing forces. On one side, as $w_{t}$ increases, each individual signal has higher variance which increases $b_{t}$. On the other side, increasing $w_{t}$ decreases the information content of the signal which decreases the weight the signal has on the posterior mean decreasing $b_{t}$. In contrast, changes in the signal correlation only affect disagreement. Increasing signal correlation causes decreasing $b_{t}$.

To link the model to uncertainty and disagreement, I rescale the posterior distribution as functions of $a_{t}, b_{t}$ and model the stochastic evolution of $a_{t}, b_{t}$ rather than $w_{t}, \tau_{t}$ over time. The posterior distribution then becomes

$$
\begin{aligned}
& s_{t} \mid\left\{z_{i, t}, a_{t}, b_{t}\right\}=\rho_{s} s_{t-1}+\frac{\sigma_{s}^{2}-a_{t}^{2}}{\sigma_{s}^{2}}\left(z_{i, t}-\rho_{s} s_{t-1}\right)+\epsilon_{i, t}^{z} \\
& \zeta_{t} \mid\left\{z_{i, t}, a_{t}, b_{t}\right\}=\rho_{s} s_{t-1}+\frac{\sigma_{s}^{2}-a_{t}^{2}-b_{t}^{2}}{\sigma_{s}^{2}-a_{t}^{2}}\left(z_{i, t}-\rho_{s} s_{t-1}\right)+\epsilon_{i, t}^{\zeta}
\end{aligned}
$$

where $\left[\epsilon_{i, t}^{z}, \epsilon_{i, t}^{\zeta}\right]^{\prime}$ is a multivariate normal with mean zero and covariance matrix

$$
\Sigma_{t}^{s, \zeta} \equiv\left[\begin{array}{cc}
a_{t}^{2} & \frac{b_{t}^{2} \sigma_{s}^{2}}{\sigma_{s}^{2}-a_{t}^{2}} \\
\frac{b_{t}^{2} \sigma_{s}^{2}}{\sigma_{s}^{2}-a_{t}^{2}} & \frac{b_{t}^{2} \sigma_{s}^{4}\left(\sigma_{s}^{2}-a_{t}^{2}-b_{t}^{2}\right)}{\left(\sigma_{s}^{2}-a_{t}^{2}\right)^{3}}
\end{array}\right]
$$

In stage $2, s_{t}$ is revealed, and each agent chooses consumption and investment. Given the signal structure, I can derive the evolution of $z_{i, t+1}$, the time $t+1$ signal for agent $i$.

$$
z_{i, t+1}=\rho_{s} s_{t}+\sigma_{s} \epsilon_{t+1}^{s}+\frac{\sigma_{s} a_{t+1}}{\sqrt{\sigma_{s}^{2}-a_{t+1}^{2}}} \epsilon_{i, t+1}^{\hat{z}}
$$

To specify the evolution for $a_{t}, b_{t}$, I note that given restrictions on $w_{t}, \tau_{t}$, I find the corre- 
sponding bounds for uncertainty and disagreement are then given by

$$
a_{t} \in\left[0, \sigma_{s}\right), \quad b_{t} \in\left[0, a_{t} \sqrt{\frac{\sigma_{s}^{2}-a_{t}^{2}}{\sigma_{s}^{2}}}\right]
$$

To keep the specification tractable, I specify the evolution of uncertainty and disagreement using an $\operatorname{AR}(1)$ process. ${ }^{3}$

$$
\begin{aligned}
& a_{t+1}=\left(1-\rho_{a}\right) \bar{a}+\rho_{a} a_{t}+\sigma_{a} \epsilon_{t+1}^{a} \\
& b_{t+1}=\left(1-\rho_{b}\right) \bar{b}+\rho_{b} b_{t}+\sigma_{b} \epsilon_{t+1}^{b}
\end{aligned}
$$

where $\left\{\epsilon_{t+1}^{a}, \epsilon_{t+1}^{b}\right\}$ are IID standard normals. In my calibration, I find the implied parameters for $\rho_{a}, \rho_{b}, \bar{a}, \bar{b}, \sigma_{a}, \sigma_{b}$ are such that $a_{t}, b_{t}$ stay within these bounds. I discuss my assumption that shocks to $a_{t}, b_{t}$ are independent more in Chapter 5 .

\subsection{Fears of Model Misspecification}

In the previous section, I captured movements in the distribution of signals using my defined concepts of uncertainty and disagreement. I then showed how uncertainty and disagreement affect each agent's first and higher order beliefs through the posterior distribution for $s_{t}, \zeta_{t}$. In this section, I add fears of model misspecification to the model.

To operationalize model ambiguity, I assume each agent fears that his posterior distribution for $s_{t}, \zeta_{t}$ in stage 1 may be misspecified. He considers alternative models by distorting the probability distributions of $\left\{\epsilon_{i, t}^{z}, \epsilon_{i, t}^{\zeta}\right\}$ in stage $1 .{ }^{4}$ The set of distortions is a family of strictly positive functions $m$ that have expectation 1 . Acting as a likelihood ratio or Radon-

3. I use the AR(1) specification for tractability. I find my results are largely unaffected if a log process is used instead.

4. I find that the results are qualitatively similar when agents also fear the evolution of the exogeneous variables $\left\{z_{i, t+1}, a_{t+1}, b_{t+1}\right\}$ may be misspecified. 
Nikodym derivative, $m$ distorts the probability distribution that the expectation is taken over in Stage 1. Let $\mathbb{E}_{i, t}, E_{i, t}$ denote the the stage 1 and stage 2 expectation operators respectively for agent $i$ at time $t$, the distorted expectation is given by

$$
\mathbb{E}_{i, t}^{m}[\cdot] \equiv \mathbb{E}_{i, t}[m(\cdot)]
$$

In order to bound the set of possibilities for $m$, the agent penalizes relative entropy or the expected log-likelihood ratio between the alternative and approximating model.

$$
\frac{1}{\theta} \mathbb{E}_{i, t}^{m}[\log m]=\frac{1}{\theta} \mathbb{E}_{i, t}[m \log m]
$$

where $1 / \theta$ acts as a Lagrange multipliers to penalize statistical deviation from the approximating model. I assume $\theta$ is a parameter common to all agents. The agent's flexibility to choose $m$ allows him to represent potentially nonparametric alternatives with the restriction that the distorted density be absolutely continuous with respect to the approximating model.

Given this constructed set of alternative models which are statistically close, I assume each agent solves a max-min problem first minimizing over his choice of distorted model then maximizing over his choice of control. The agent's choices are then robust to a worst case model where the agent selects the form of misspecification with the largest utility consequences. The distortion affects the distribution of $s_{t}, \zeta_{t}$, and thus causes deviations in the posterior distribution of agents away from the original baseline specification. I calibrate $\theta$ such that the worst case model that each agent selects is statistically plausible when compared to the undistorted model. I provide more details on the calibration of $\theta$ in Chapter 6. 


\subsection{Equilibrium and Value Function}

Due to the incomplete information and complementarity of goods, each agent's choice of optimal labor and consumption depend on his beliefs about unknown productivity as well as his beliefs about what other people believe about productivity. From the resource constraint for each agent, note that aggregate variables enter each individual problem through the intermediate goods price $p_{i, t}$. I denote aggregate labor, consumption, capital stock, and output as

$$
N_{t}=\int_{0}^{1} n_{i, t} d i, \quad C_{t}=\int_{0}^{1} c_{i, t} d i, \quad K_{t}=\int_{0}^{1} k_{i, t} d i, \quad Y_{t}=\exp \int_{0}^{1} \log y_{i, t} d i
$$

To solve the model, I search for a recursive equilibrium in which each agent optimally chooses labor and consumption taking an intermediate good price law and aggregate capital law of motion as given. I then look for a fixed point where the price law and aggregate law of motion are consistent with the beliefs and choices of each agent.

Due to the fact that $\theta, a_{t}, b_{t}$ are common to all agents, each agent's Hamilton-JacobiBellman (HJB) equation will have the same form. To simplify notation, I drop the $i, t$ subscripts below and use $(\cdot)^{\prime},(\cdot)^{-}$to denote next and previous period variables respectively. Once again I let $\mathbb{E}[\cdot]$ denote the expectation in stage 1 when $s, \zeta$ are unknown, and $E[\cdot]$ denote the expectation in stage 2 when $z^{\prime}, a^{\prime}, b^{\prime}$ are unknown.

\subsubsection{Recursive Equilibrium}

More formally I define a recursive equilibrium as the quartet $\left\{P, G, V^{1}, V^{2}\right\}$ such that

- Treating the intermediate goods price $P$ and the law of motion for the aggregate capital stock $G$ as exogeneous, each agent solves for his value function in stage 1 and 2, labeled $V^{1}, V^{2}$ respectively, to optimally choose labor, consumption, and worst case model. 


$$
\begin{aligned}
V^{1}\left(k, z ; s^{-}, K, a, b\right) & =\max _{n} \min _{\substack{m>0 \\
\mathbb{E}[m]=1}}-\frac{n^{1+\nu}}{1+\nu}+\mathbb{E}\left[m V^{2}\left(u ; s^{-}, K, s, \zeta, a, b\right)\right]+\frac{1}{\theta} \mathbb{E}[m \log (m)] \\
u & =P \exp (s) k^{\alpha} n^{1-\alpha}+(1-\delta) k \\
V^{2}\left(u ; s^{-}, K, s, \zeta, a, b\right) & =\max _{c} \frac{c^{1-\gamma}}{1-\gamma}+\beta E\left[V^{1}\left(k^{\prime}, z^{\prime} ; s, K^{\prime}, a^{\prime}, b^{\prime}\right)\right] \\
k^{\prime} & =u-c \\
K^{\prime} & =G
\end{aligned}
$$

- Given an agent's optimal choices, the assumed intermediate goods price is given by $P=\frac{Y}{\exp (s) k^{\alpha} n^{1-\alpha}}$ consistent with the Cobb-Douglas aggregation into a final good.

- Given an agent's optimal choices, the assumed law of motion for the aggregate capital stock is given by $G=Y+(1-\delta) K-C$ consistent with taking the integral over individual agent's capital.

In stage 1 , each agent takes the intermediate goods price $P$ as exogeneous. He first minimizes over his choice of worst case model. Parameterized by $m$, this log-likelihood ratio represents a distortion to the joint distribution of $\left[s_{t}, \zeta_{t}\right]^{\prime}$. The agent picks the worst case distortion $m^{*}$ which has the largest utility consequences bounded by the penalty term. He then chooses labor and commits to a level of production $u$ under the distorted measure.

In stage $2, s, \zeta$ are revealed, and production occurs. Taking the aggregate law of motion for $G$ as given, each agent then chooses consumption and investment taking expectations over next periods signal, uncertainty, and disagreement $\left(z^{\prime}, a^{\prime}, b^{\prime}\right)$.

Solving the FOC for each agent's choice of optimal distortion $m^{*}$, I find

$$
m^{*}=\exp \left(-\theta V^{2}\right) / \mathbb{E}\left[\exp \left(-\theta V^{2}\right)\right]
$$

Note the model misspecification fears of the agent lead to exponential tilt probabilities away 
from the original model. He puts increased probability weights on the outcomes of $s_{t}, \zeta_{t}$ that lead to a lower value function in stage 2 . The expectation in the denominator normalizes $m^{*}$ to have expectation 1 and remain viable as a Radon-Nikodym derivative. Plugging in the optimal distortion, the HJB becomes

$$
\begin{aligned}
V^{1}\left(k, z ; s^{-}, K, a, b\right) & =\max _{n}-\frac{n^{1+\nu}}{1+\nu}-\frac{1}{\theta} \log \mathbb{E}\left[\exp \left(-\theta V^{2}\left(u ; s^{-}, K, s, \zeta, a, b\right)\right)\right] \\
V^{2}\left(u ; s^{-}, K, s, \zeta, a, b\right) & =\max _{c} \frac{c^{1-\gamma}}{1-\gamma}+\beta E\left[V^{1}\left(k^{\prime}, z^{\prime} ; s, K^{\prime}, a^{\prime}, b^{\prime}\right)\right]
\end{aligned}
$$

The fears of model misspecification introduce a $\log \mathbb{E} \exp (\cdot)$ certainty equivalent term which increases the curvature in the value function. As a result, the fears of model misspecification cause the agent to be more cautious about dispersion in $s_{t}, \zeta_{t}$. Changes in uncertainty and disagreement affect the posterior variance of $s_{t}, \zeta_{t}$ and through the continuation value, affect each agent's choice of controls. Solving for optimal labor and consumption and applying the envelope condition, I find the FOCs for labor and consumption become

$$
\begin{aligned}
n_{i, t}^{\nu} & =\mathbb{E}_{i, t}\left[m_{i, t}^{*}(1-\alpha) p_{i, t} \exp \left(s_{t}\right) k_{i, t}^{\alpha} n_{i, t}^{-\alpha} c_{i, t}^{-\gamma}\right] \\
c_{i, t}^{-\gamma} & =\beta E_{i, t}\left[\mathbb{E}_{i, t+1}\left[m_{i, t+1}^{*} c_{i, t+1}^{-\gamma}\left\{\alpha p_{i, t+1} \exp \left(s_{t+1}\right) k_{i, t+1}^{\alpha-1} n_{i, t+1}^{1-\alpha}+(1-\delta)\right\}\right]\right]
\end{aligned}
$$

where the expectations for the optimal controls are taken under the distorted measure $m^{*}$. 


\section{CHAPTER 3 \\ SOLUTION METHOD}

In the previous section, I assumed first and higher order beliefs in the model could be summarized using the posterior distribution for $s_{t}, \zeta_{t}$. I now describe my solution method and verify this assumption holds.

\subsection{Data Generating Process}

Since agents have fears of model misspecification, I must pick a data generating process to simulate and analyze the model and implications. In this paper, I assume that under the data generating process, at each time $t, z_{i, t}=z_{j, t}$ for all $i, j \in[0,1]$. My assumption implies that agent's beliefs about the signal structure across agents differs from the data generating process, and they do not update their beliefs when the actions of all other agents is revealed in stage 2. Every agent believes there is correlation $\tau_{t}$ across the signals and a disagreement of $b_{t}$ but under the data generating process, $\tau_{t}=1$ and $b_{t}=0$ for all $t$. This assumption while strong allows each individual capital stock to remain equal and thus allows me to avoid keeping track of the full distribution of capital across agents as a state variable. As a result, the behavior of agents in this setting will still be dependent on what the agents believe about the distribution of signals but will not capture the effects of the distribution of capital on each agent's decision. While these effects are interesting, my paper focuses on the how the distribution of signals affects agents decisions through the channel of first and higher order beliefs rather than heterogeneity in capital.

In addition, I assume that the baseline evolution for log aggregate productivity $s_{t}$ and the baseline distribution of the private signal $z_{i, t}$ relative to $s_{t}$ are correctly specified. Each agent

thus receives the same signal but the signal has noise $w_{t} \epsilon_{i, t}^{\hat{z}}$ which is distributed normally. The productivity under the data generating process follows the $\mathrm{AR}(1)$ specification that 
coincides with agents' undistorted baseline beliefs about $s_{t}$. A potential avenue of extension would be to consider how the results change given the agent's evolution of $s_{t}, z_{i, t}$ are also misspecified. However, I do not consider it here since I rule out learning and focus on the effects coming from first and higher order beliefs.

\subsection{Optimal Controls}

To distinguish the information sets available at stage 1 and 2 in my model, I separate the state variables

$$
\begin{aligned}
& x_{i, t}^{1}=\left\{k_{i, t}, K_{t}, z_{i, t}, a_{t}, b_{t}\right\}, \quad w_{i, t}^{1}=\left\{\epsilon_{i, t}^{z}, \epsilon_{i, t}^{\zeta}\right\} \\
& x_{i, t}^{2}=\left\{s_{t}, \zeta_{t}\right\}, \quad w_{i, t+1}^{2}=\left\{\epsilon_{i, t+1}^{\hat{s}}, \epsilon_{t+1}^{a}, \epsilon_{t+1}^{b}\right\}
\end{aligned}
$$

I then propose that each agent's choice of optimal labor and consumption can be written as functions

$$
n_{t}=n\left(x_{i, t}^{1}, x_{i, t-1}^{2}\right), \quad c_{t}=c\left(x_{i, t}^{1}, x_{i, t-1}^{2}, x_{i, t}^{2}\right)
$$

In stage 1 of time $t, x_{i, t}^{1}$ represents the individual and aggregate capital stock, the private signal, uncertainty, and disagreement. These state variables affect the an agent's choice through the marginal product of labor and the posterior distribution for $s_{t}, \zeta_{t} \cdot x_{i, t-1}^{2}$ represents the previous period's productivity and average signal. Since, the agent's private signal is subject to error, each agent uses the previous period's productivity $s_{t-1}$ to measure the surprise in his signal, an important part of the posterior for $s_{t}, \zeta_{t}$ as shown in the previous section. The agent then chooses labor based on $\left\{x_{i, t}^{1}, x_{i, t-1}^{2}\right\}$ where $w_{i, t}^{1}$ represent the unknown stochastic parts of $s_{t}, \zeta_{t}$.

In stage 2 of time $t, x_{i, t}^{2}$ is revealed to the agent since the productivity and choices of 
all other agents are revealed. The agent then chooses consumption and investment with his available information set being $\left\{x_{i, t}^{1}, x_{i, t}^{2}, x_{i, t-1}^{2}\right\}$. He forms expectations about $x_{i, t+1}^{1}$ where $w_{i, t+1}^{2}$ represents the unknown stochastic parts of $z_{i, t+1}, a_{t+1}, b_{t+1}$. The evolution of the local and aggregate capital stock are known since each agent picks his own consumption and assumes an exogeneous law of motion for the aggregate capital stock.

Given my proposed policy rule for individual labor and consumption, I can prove $s_{t}, \zeta_{t}$ are sufficient to capture the beliefs of each agent by showing that my proposed form of controls satisfies the conditions for a recursive equilibrium. Since the form of control is the same for all agents, I drop the $i$ subscripts going forward for notational convenience.

\subsection{Perturbation Method}

To solve the model, I use a first order approximation to a perturbation around the nonstochastic steady state. I extend the method used in [3] which is based on [15] and [5]. Consider a scalar perturbation parameter $q$ which scales the random shocks in my economy. Under standard regularity conditions, I can then write the evolution of the state variables under the proposed control law as

$$
\begin{aligned}
x_{t}^{2} & =X\left(x_{t}^{1}(q), x_{t-1}^{2}(q), q w_{t}^{1}, q\right) \\
x_{t+1}^{1} & =Y\left(x_{t}^{1}(q), x_{t-1}^{2}(q), x_{t}^{2}(q), q w_{t+1}^{2}, q\right)
\end{aligned}
$$

The function $X$ captures the posterior distribution for $s_{t}, \zeta_{t}$. The function $Y$ captures the distribution of next period's signal, uncertainty, and disagreement as well as the evolution of next periods local and aggregate capital stock. Taking a first order expansion around the 
non-stochastic steady state, I can approximate

$$
\begin{aligned}
x_{1, t}^{2} & =X_{x^{1}} x_{1, t}^{1}+X_{x^{2}-} x_{1, t-1}^{2}+X_{w^{1}} w_{t}^{1}+X_{q} \\
x_{1, t+1}^{1} & =Y_{x^{1}} x_{1, t}^{1}+Y_{x^{2}-} x_{1, t-1}^{2}+Y_{x^{2}} x_{1, t}^{2}+Y_{w^{2}+} w_{t+1}^{2}+Y_{q}
\end{aligned}
$$

I let $x_{1, t}^{2}, x_{1, t+1}^{1}$ denote the first order expansion of $x_{t}^{2}, x_{t+1}^{1}$ respectively. The coefficients $X_{x^{1}}, X_{x^{2}-}, X_{w^{1}}, X_{q}$ represent derivatives of $X$ evaluated at the steady state and $q=0 . \mathrm{I}$ define $Y_{x^{1}}, Y_{x^{2}-}, Y_{x^{2}}, Y_{w^{2}+}, Y_{q}$ analogously.

Using the same notation and perturbation, I approximate the agent's choice of controls under the proposed control law

$$
\begin{aligned}
& n_{1, t}=n_{x^{1}} x_{1, t}^{1}+n_{x^{2}-} x_{1, t-1}^{2}+n_{q} \\
& c_{1, t}=c_{x^{1}} x_{1, t}^{1}+c_{x^{2}-} x_{1, t-1}^{2}+c_{x^{2}} x_{1, t}^{2}+c_{q}
\end{aligned}
$$

Note that the coefficients $\left(n_{x^{1}}, n_{x^{2}-}, n_{q}, c_{x^{1}}, c_{x^{2}-}, c_{x^{2}}, c_{q}\right)$ are functions of the steady state and thus the same for all agents. Taking an integral over agents $i$ then allows me to write the aggregate variables consistent with each individual's decision as

$$
\begin{aligned}
& N_{1, t}=n_{x^{1}}\left[A_{x^{1}} x_{1, t}^{1}+A_{x^{2}} x_{1, t}^{2}\right]+n_{x^{2}-} x_{1, t-1}^{2}+n_{q} \\
& C_{1, t}=c_{x^{1}}\left[A_{x^{1}} x_{1, t}^{1}+A_{x^{2}} x_{1, t}^{2}\right]+c_{x^{2}-} x_{1, t-1}^{2}+c_{x^{2}} x_{1, t}^{2}+c_{q}
\end{aligned}
$$

where $A_{x^{1}}, A_{x^{2}}$ are matrices which transform the individual's $x_{1, t}^{1}=\left\{k_{1, t}, K_{1, t}, z_{1, t}, a_{1, t}, b_{1, t}\right\}$ to the corresponding aggregate quantities $\left\{K_{1, t}, K_{1, t}, \zeta_{1, t}, a_{1, t}, b_{1, t}\right\}$.

Looking at the FOCs for each agent from Equations (2.33) and (2.34), I note that the continuation value matters for the decisions of each agent through the choice of optimal worst case model. I approximate the HJB equation of each agent using the following innovation 
to the perturbation from [3].

$$
\begin{aligned}
& V_{t}^{1}(q)=u^{1}\left(n_{t}(q), q\right)-\frac{q}{\theta} \log \mathbb{E}_{t}\left[\exp \left(-\frac{\theta}{q} V_{t}^{2}(q)\right)\right] \\
& V_{t}^{2}(q)=u^{2}\left(c_{t}(q), q\right)+\beta E_{t}\left[V_{t+1}^{1}(q)\right]
\end{aligned}
$$

This unique perturbation on the certainty equivalent is necessary since, if I follow the standard perturbation, the exponent in Equation (3.12) and the numerator in Equation (2.30) becomes

$$
-\theta V_{t}^{2}(q) \approx-\theta\left(\overline{\left(V^{2}\right)}+q V_{1, t}^{2}\right)
$$

where $\overline{\left(V^{2}\right)}$ represents the steady state value of $V^{2}$. Note that as $q \rightarrow 0$, the belief distortion in the model vanishes. In the steady state, since the economy is deterministic, there is no fear of model misspecification. Each agent's decision to first order as a result will not be affected by model ambiguity under the standard perturbation. Expanding the HJB equation with the modified perturbation gives me

$$
\begin{aligned}
& V_{1, t}^{1}=u_{1, t}^{1}-\frac{1}{\theta} \log \mathbb{E}_{t}\left[\exp \left(-\theta V_{1, t}^{2}\right)\right] \\
& V_{1, t}^{2}=u_{1, t}^{2}+\beta E_{t}\left[V_{1, t+1}^{1}\right]
\end{aligned}
$$

The first order expansion to the value function under my proposed control laws (3.8)-(3.9) has the form

$$
\begin{aligned}
& V_{1, t}^{1}=V_{q}^{1}+V_{x^{1}}^{1} x_{1, t}^{1}+V_{x^{2}-}^{1} x_{1, t-1}^{2} \\
& V_{1, t}^{2}=V_{q}^{2}+V_{x^{1}}^{2} x_{1, t}^{1}+V_{x^{2}-}^{2} x_{1, t-1}^{2}+V_{x^{2}}^{2} x_{1, t}^{2}
\end{aligned}
$$

I note that the worst case distortion $m_{t}^{*}$ does not vanish as $q \rightarrow 0$ under the new perturbation. 
Expanding Equation (2.30), I find to zeroth order

$$
m_{0, t}^{*}=\exp \left(-\theta V_{1, t}^{2}\right) / \mathbb{E}_{t}\left[\exp \left(-\theta V_{1, t}^{2}\right)\right]
$$

The distortion $m_{0, t}^{*}$ applied to the random shock $w_{t}^{1}$ then implies the distribution under the distorted measure is

$$
w_{t}^{1} \sim \mathcal{N}\left(-\theta V_{x^{2}}^{2} X_{w^{1}} \Sigma_{t}^{s, \zeta}, \Sigma_{t}^{s, \zeta}\right)
$$

Note that the fears of model misspecification impart a mean shift to the random variables in the model. The magnitude of the mean distortion is proportional to the degree of fears of model misspecification, the value function derivative, and covariance matrix of the random shocks. Thus the agent shifts the mean of the distribution of $s_{t}, \zeta_{t}$ in the direction which is worst for his continuation value. He balances the relative shifts across $s_{t}, \zeta_{t}$ based on their relative volatility as well as their relative marginal contribution to the continuation value. $\Sigma_{t}^{s, \zeta}$ is a non-linear function of uncertainty and disagreement as defined in Equation (2.17) which implies that the resulting control law will depend on $a_{t}, b_{t}$ under fears of potential model misspecification. To maintain the linear structure, I take a first order expansion to the covariance matrix $\Sigma_{t}^{s, \zeta}$.

$$
\Sigma_{t}^{s, \zeta} \approx \bar{\Sigma}_{s, \zeta}+\Sigma_{x^{1}}^{s, \zeta} x_{1, t}^{1}
$$

where $\bar{\Sigma}_{s, \zeta}$ represents the steady state value and $\Sigma_{x^{1}}^{s, \zeta}$ represents the element-wise derivative of the covariance matrix w.r.t. $x_{t}^{1}$ evaluated at the steady state. This approximation allows me to capture the role of the dynamics of uncertainty and disagreement on the covariance matrix and the agent's choice of controls. I ensure the approximation to the covariance matrix remains positive semi-definite under simulated values for $a_{t}, b_{t}$, redrawing values of 
$a_{t}, b_{t}$ if the condition is violated. I find that under my calibrated evolution for $a_{t}, b_{t}$ this happens rarely.

I now turn to approximating the FOCs. For notational convenience, let define $e_{t}, h_{t+1}$

$$
\begin{aligned}
e_{t} & =(1-\alpha) p_{i, t} \exp \left(s_{t}\right) k_{i, t}^{\alpha} n_{i, t}^{-\alpha} c_{i, t}^{-\gamma}-n_{i, t}^{\nu} \\
h_{t+1} & =\beta c_{i, t+1}^{-\gamma}\left\{\alpha p_{i, t+1} \exp \left(s_{t+1}\right) k_{i, t+1}^{\alpha-1} n_{i, t+1}^{1-\alpha}+(1-\delta)\right\}-c_{i, t}^{-\gamma}
\end{aligned}
$$

Rewriting the FOCs given in Equations (2.33) and (2.34) and expanding to first order I find

$$
\begin{aligned}
0 & =\mathbb{E}_{t}\left[m_{t}^{*} e_{t}\right] \approx \mathbb{E}_{t}\left[m_{0, t}^{*} e_{1, t}\right]+\mathbb{E}_{t}\left[m_{1, t}^{*} e_{0, t}\right]=\mathbb{E}_{t}\left[m_{0, t}^{*} e_{1, t}\right] \\
0 & =E_{t}\left[\mathbb{E}_{t+1}\left[m_{t+1}^{*} h_{t+1}\right]\right] \approx E_{t}\left[\mathbb{E}_{t+1}\left[m_{0, t+1}^{*} h_{1, t+1}\right]\right]+E_{t}\left[\mathbb{E}_{t+1}\left[m_{1, t+1}^{*} h_{0, t+1}\right]\right] \\
& =E_{t}\left[\mathbb{E}_{t+1}\left[m_{0, t+1}^{*} h_{1, t+1}\right]\right]
\end{aligned}
$$

where I utilized the fact that the zeroth order expansion of both FOCs is a constant 0 since I am using non-stochastic steady state values.

To ensure that my recursive equilibrium is consistent, I plug in my desired forms for $P, G$ as functions of the aggregate controls. Note that under the data generating process, $Y_{t}=y_{i, t}$ for all $i$. I find $e_{t}, h_{t+1}$ are functions of the form

$$
\begin{aligned}
e_{t} & =e\left(n_{t}, c_{t}, N_{t}, x_{t}^{1}, x_{t}^{2}, w_{t}^{1}\right) \\
h_{t+1} & =h\left(c_{t+1}, c_{t}, N_{t+1}, N_{t}, C_{t}, x_{t+1}^{1}, x_{t+1}^{2}, x_{t}^{1}, x_{t}^{2}, w_{t+1}^{1}, w_{t+1}^{2}\right)
\end{aligned}
$$

Taking a first order expansion of the FOCs then gives me a set of linear equations involving the state variables $\left\{x_{t}^{1}, x_{t}^{2}\right\}$, controls $\left\{n_{t}, c_{t}, N_{t}, C_{t}\right\}$, continuation values $\left\{V_{t}^{1}, V_{t}^{2}\right\}$, and stochastic variables $\left\{w_{t}^{1}, w_{t+1}^{2}\right\}$. As noted above, the aggregate controls can be written as linear functions of the individual controls. The continuation values and the expectation of $w_{t}^{1}, w_{t+1}^{1}$ under the distorted measure can be written as linear functions of the state variables. 
Thus I find the FOCs can be written as a system of linear equations with the coefficients on the first order expansions of $X_{t}, Y_{t}, n_{t}, c_{t}$ as the unknowns.

By plugging in the functional forms for $P, G$, and solving under the restrictions that $n_{t}, c_{t}$ imply for $N_{t}, C_{t}$, I ensure any solution to the coefficients I find is consistent with a recursive equilibrium. Since I have found everything can be written as a function of my state variables given my proposed control law, I verify my assumption that the posterior distribution for $s_{t}, \zeta_{t}$ is sufficient for capturing the beliefs of each agent in Stage 1. 


\section{CHAPTER 4}

\section{LINKING UNCERTAINTY AND DISAGREEMENT TO DATA}

In the previous sections, I discussed how my defined concepts of uncertainty and disagreement capture movements in the distribution of signals that agents receive about productivity. However, empirically it is difficult to tell which sources of information agents use as signals. Rather than calibrating my model using assumptions about which signals agents use, I compute implied forecasts that my agents would make for the growth rate of real GDP under uncertainty and disagreement. In this way, I can infer from the time-series properties of forecasts, the time-series properties of uncertainty and disagreement.

Let $r_{t}$ denote the real GDP growth rate in my model. I find then

$$
r_{t}=\frac{\exp \left(s_{t}\right) K_{t}^{\alpha} N_{t}^{1-\alpha}}{\exp \left(s_{t-1}\right) K_{t-1}^{\alpha} N_{t-1}^{1-\alpha}}-1
$$

In Stage 1 of time period $t$, each agent's expected real GDP growth rate then depends on the previous periods aggregate labor choice $N_{t-1}$ and aggregate investment decision $K_{t}, K_{t-1}$ which are known as well as the expectations about the unknown variables $s_{t}, N_{t}$. For tractability, I approximate $r_{t}$ to first order using the simplification

$$
r_{1, t} \approx s_{1, t}
$$

This approximation assumes that the system is in steady state at $t-1$ and that each agent does not take into account the changes in aggregate labor when predicting the growth rate of real GDP.

The assumption at $t-1$ simplifies the state dependence for the forecast of the growth rate, so I can avoid having to measure changes in the productive capital stock which has been a difficult problem in the literature. I assume that agents do not take into account the complementarity effect of their expectations on aggregate labor for tractability. In my model, 
agents take a price law as given and internalize the effect of the distribution of productivity on aggregate labor through the exogenous price law. However, I show in Appendix $\mathrm{C}$ that taking changes in aggregate labor into account actually hinders my ability to fit dynamics in the forecast of $r_{t}$ using uncertainty and disagreement. This result suggests either survey participants do not accurately take into account the effect on aggregate labor when making forecasts or that structure of my model is not rich enough to capture all the dynamics. I leave adding richer dynamics to my model as an area of future research, and use the simplified assumption in Equation (4.2). To support the use of the approximation, I run additional tests comparing TFP to the real GDP growth rate in Appendix C.

Given this simplification, I find that then that my signal structure implies a distribution for the forecast of real GDP growth rate which is normal. Given normality, uncertainty and disagreement can be calibrated by taking the corresponding moments in the posterior distribution of the forecast for growth rate. Note that my definitions of uncertainty and disagreement are independent of whether expectations are taken under the undistorted or distorted measure. From Equation (3.20), I find that the distortion adds a mean shift to the distribution of $s_{t}$. However, this mean shift is a function of the covariance matrix $\Sigma_{t}^{s, \zeta}$ which is a function of $a_{t}, b_{t}$ and does not depend on $z_{i, t}$. As a result, disagreement which takes the standard deviation across agents would be unaffected since $a_{t}, b_{t}$ are common to all agents. The distortion does not affect the variance of $s_{t}, \zeta_{t}$, and as a result, the expression for uncertainty which takes the variance of $s_{t}$ is also unaffected whether the undistorted or distorted densities are used. 


\section{CHAPTER 5 SURVEY EVIDENCE}

The Survey of Professional Forecasters (SPF) is a quarterly survey of macroeconomic forecasters in the United States that was started in 1968. The survey is mailed four times a year on the day after the release of the advance reports of the National Income and Product Accounts (NIPA) data for the preceding quarter. In addition to point forecasts for a range of variables at varying horizons, the SPF asks for density forecasts for variables such as aggregate output and inflation. The question for aggregate output was switched from nominal GDP to real GNP in 1981 and switched again from real GNP to real GDP in 1992 which shortens the available sample of data. I focus on both point and density forecasts for the growth rate from 1992Q1 to 2017Q4, as I am interested in the comovements of real variables with beliefs about economic conditions. ${ }^{1}$

For density forecasts, the survey asks forecasters to assign a probability to each of a number of intervals, or bins, in which the growth rate of aggregate output might fall, in the current year and in the next year. The definition of growth rate is annual-average over annual-average which is defined as the percent change in the annual-average level from one year to the next. The probability estimates are for fixed event forecasts: in each quarterly survey of a given calendar year, the participants provide their probability estimates for annual-average over annual-average growth in the current year (the year in which the survey was conducted) and the following year. More formally, let $Y_{t, 1}, Y_{t, 2}, Y_{t, 3}, Y_{t, 4}$ be the real GDP in each of the four quarters in year $t$. For each quarterly survey in year $t$, the SPF

1. While one can approximate that the growth rate of real GNP is representative of real GDP growth from 1981 to 1992, I show in Appendix B that there is a discontinuity in the properties of the density forecasts before and after 1992 . 
asks participants for a density forecast of

$$
x_{t} \equiv \frac{\frac{1}{4}\left(Y_{t, 1}+Y_{t, 2}+Y_{t, 3}+Y_{t, 4}\right)}{\frac{1}{4}\left(Y_{t-1,1}+Y_{t-1,2}+Y_{t-1,3}+Y_{t-1,4}\right)}-1
$$

Thus, in the survey conducted in quarter 1 of year t, $Y_{t, 1}, Y_{t, 2}, Y_{t, 3}, Y_{t, 4}$ are all unknown for respondents to predict, but for the survey conducted in quarter 4 of year t, only $Y_{t, 4}$ is unknown.

For point forecasts, the survey asks participants to predict the level of GDP for each quarter going four quarters forward as well as the annual average level of GDP for the current and next calendar year. The survey provides the advance estimates for the level of GDP as well as the GDP price index for the previous quarter and year when asking for the forecasts. In order to make the point forecasts comparable to the growth rates predicted in the density forecasts, I convert the point predictions of levels into point predictions of percentages. I then compute the implied annual-average over annual-average growth rate.

Since the previous year's GDP level is provided to respondents, I focus on predictions for the current calendar year and calculate the percentage change between the forecast and the previous year's value.

The structure and timing of the survey introduces a number of complications in the data:

a) The number of respondents varies over time with a minimum of 23 to a maximum of 47 .

b) The number of bins and their ranges have changed over time. From 1992:Q1 to 2009:Q1 there were 10 bins ranging from $-2 \%$ to 6\%. From 2009:Q2 to the present there were 11 bins ranging from $-3 \%$ to $6 \%$.

c) The Q1 forecast for the growth rate has the advance estimates from NIPA up to Q4 for the previous year while the Q4 forecast for the growth rate has advance estimates from NIPA up to Q3 for the current year. 
I assume a) and b) are minor and inconsequential given the the minimum number of respondents and the number of bins. In order to address concerns with c), I specify a choice of quarter to analyze in each year.

\subsection{Constructing Probability Densities}

The survey data gives me a discrete estimate of the cumulative distribution function (CDF) for each participant. I index participants $j \in\left\{1, \ldots, J_{t}\right\}$ where $J_{t}$ is the total number of participants at time $t$ and index bins $\omega \in\left\{1, \ldots \Omega_{t}\right\}$ where $\Omega_{t}$ is the total number of bins at time $t$. Let bin $\omega$ correspond to the interval $\left[u_{\omega}^{\ell}, u_{\omega}^{u}\right)$ and $\operatorname{Pr}_{j, t}(\omega)$ denote the response of participant $j$ at time $t$ to the probability that the growth rate lies in bin $\omega$. For the end bins $\left(k=\omega\right.$ and $\left.k=\Omega_{t}\right)$ which are open intervals in the survey, I assume they have the same width as all the other bins. In order to construct a probability density function (PDF), I make the assumption about that the distribution of the PDF within each of the bins is uniform. This assumption then allows me to integrate the PDF to compute the mean

and standard deviation $\left(\mu_{t \mid o}^{j}, \sigma_{t \mid o}^{j}\right)$ of the distribution in quarter $o$ of each year $t$ for each participant $j$.

My assumption of a uniform distribution within the bins strikes a balance between placing a restrictive parametric form of the distribution and flexibility to fit the wide variety of responses from the survey data. In Appendix B, I explore fitting a normal and a generalized beta distribution to the data to ensure my results are robust to other distributional forms as well as potential truncation in the first and last bins.

\subsection{Summary Statistics of Forecasts}

Following the methodology of [7], I construct forecast errors (FE) and forecast revisions (FR) using both the point and the mean of the density estimates for the growth rate of 
real GDP to ensure survey responses are consistent and accurate. Let $x_{\left.t\right|_{o}}^{j}$ denote the point forecast for the real GDP growth rate $x_{t}$ made in quarter $o$ of year $t$. I define the forecast error and revision as

$$
\begin{gathered}
(\text { Point FE) })_{\left.t\right|_{o}}^{j}=x_{t}-x_{\left.t\right|_{o}}^{j} \\
(\text { Point FR })_{\left.t\right|_{o}}^{j}=x_{\left.t\right|_{o}}^{j}-x_{\left.t\right|_{o}-1}^{j} \\
(\text { Density FE })_{\left.t\right|_{o}}^{j}=x_{t}-\mu_{\left.t\right|_{o}}^{j} \\
(\text { Density FR })_{\left.t\right|_{o}}^{j}=\mu_{\left.t\right|_{o}}^{j}-\mu_{\left.t\right|_{o}-1}^{j}
\end{gathered}
$$

Since the density estimates correspond to the annual-average over annual-average level, I construct the forecast revision using the change in forecast between each consecutive quarter within each calendar year. This method gives me three potential forecast revisions (differences between quarter 1-2, 2-3, and 3-4).

To ensure my results are comparable, I eliminate data points in which survey respondents only provide a point or only provide a density forecast. I also eliminate data points in which I cannot compute the forecast revision as the respondent did not participate for two consecutive quarters. Lastly, I eliminate respondents who appear less than 3 times in the sample.

I list a table of summary statistics comparing forecast errors and forecaster revisions for density and point forecasts for the growth rate of real GDP in Table 5.1. Note that the data show that on average participants of the SPF do a good job forecasting real GDP growth. The mean error for both density and point forecasts are less than 13 basis points for predictions made in the second, third, or fourth quarters. I find the mean forecast revisions for both density and point forecasts are smaller than 22 basis points in absolute value. This suggests forecasters do not systematically adjust their forecasts in one direction or another direction in response to new information. I also find sizable dispersion in the forecast across 


\begin{tabular}{c|cc|ccccccc|cccccc}
\hline \multicolumn{4}{c}{} & \multicolumn{1}{c}{ Actual } & \multicolumn{1}{c}{ Forecast } & \multicolumn{2}{c}{ Eensity } & \multicolumn{1}{c}{ Errors } & \multicolumn{4}{c}{ Revisions } & \multicolumn{4}{c}{ Forecast } & \multicolumn{2}{c}{ Errors } & \multicolumn{2}{c}{ Revisions } \\
Quarter & Mean & SD & Mean & SD & Mean & SD & Mean & SD & Mean & SD & Mean & SD & Mean & SD \\
\hline 4 & & & 2.50 & 1.33 & -0.07 & 0.80 & 0.11 & 0.51 & 2.34 & 1.41 & 0.09 & 0.74 & -0.15 & 0.68 \\
3 & 2.53 & 1.61 & 2.36 & 1.30 & 0.03 & 0.81 & -0.13 & 0.52 & 2.47 & 1.10 & -0.08 & 0.80 & 0.10 & 0.66 \\
2 & & & 2.45 & 1.34 & -0.13 & 0.86 & 0.01 & 0.63 & 2.36 & 1.22 & -0.05 & 0.98 & -0.22 & 0.76 \\
\hline
\end{tabular}

Table 5.1: Summary statistics for the forecasts, forecast errors, and forecast revisions for both the density and point estimates for real GDP. Forecast revisions are computed using the forecast in the previous quarter. The SD for the density forecast is the standard deviation across forecasters of the fitted mean of the density estimate. All values annualized and in percent.

forecasters suggesting that there is a lack of consensus among forecasters both for the point prediction and the mean of the density prediction.

The summary statistics also suggest no large discrepancies in the mean and standard deviation of the forecasts, errors, and revisions between the density and point forecasts. The fact that forecasters do a good job in predicting the growth rate is consistent with my assumption that survey respondents provide the undistorted distribution in their responses. I also find that respondents use an average of 2.95 bins in their density responses and on average allocate $.28 \%$ of probability weight in the end bins. The number of bins used indicates that respondents understand what a probability density represents, and the data can be a source for understanding dispersion within respondents densities. The minimal weight in the end bins suggests truncation does not play a major role in the analysis. I take these results as evidence that using SPF density forecasts to extract uncertainty and disagreement is reasonable.

\subsection{Constructing Uncertainty and Disagreement}

To construct uncertainty and disagreement, I use density forecasts made in a specific quarter of each calendar year. I verify that my results are qualitatively similar across the choice of quarter. Given a choice of quarter, I construct estimates at each time $t$ by taking 
sample moments across participants $j$ for the survey taken in quarter $o$ of each year. I define the estimators

$$
\begin{aligned}
\hat{\psi}_{t \mid o} & \equiv \frac{1}{J_{t}} \sum_{j=1}^{J_{t}} \mu_{t \mid o}^{j} \\
\hat{\chi}_{t \mid o} & \equiv \frac{1}{J_{t}} \sum_{j=1}^{J_{t}} \sigma_{t \mid o}^{j} \\
\hat{\xi}_{t \mid o} & \equiv\left\{\frac{1}{J_{t}-1} \sum_{j=1}^{J_{t}}\left[\mu_{t \mid o}^{j}-\frac{1}{J_{t}} \sum_{j=1}^{J_{t}} \mu_{t \mid o}^{j}\right]^{2}\right\}^{1 / 2}
\end{aligned}
$$

Note that $\hat{\psi}_{t \mid o}$ represents the average prediction across agents for the mean predicted growth rate. $\hat{\chi}_{t \mid o}$ represents the average across agents $j$ of each agent's standard deviation for the distribution of the growth rate. Thus it represents a measure of the average dispersion in each agents' individual distributions for the growth rate. In the data, I find that $\sigma_{t \mid o}^{j}$ varies across agents $j$. However, since my model does not capture this heterogeneity, I take an average of agents to create a representative value consistent with my definition of uncertainty. The estimator $\hat{\xi}_{\left.t\right|_{o}}$ represents the sample standard deviation across agents of each agent's mean predicted growth rate. Thus $\hat{\xi}_{t \mid o}$ measures the dispersion across agents of what they predicted as the mean growth rate consistent with my definition of disagreement. I plot $\hat{\psi}_{t \mid o}, \hat{\chi}_{t \mid o}, \hat{\xi}_{t \mid o}$ setting $o=4$ in Figure 5.1.

Note that $\hat{\chi}_{t \mid o}, \hat{\xi}_{t \mid o}$ have very different time-series properties. I find $\hat{\chi}_{t \mid o}$ seems to be relatively constant over time and unaffected by recession periods. $\hat{\xi}_{t \mid o}$ varies more over time and has a big spike during the Financial Crisis. Interpreting the plots, I find that individual forecasters adjust their mean predicted growth rate depending on economic conditions, but forecasters do not shift the variance of their predicted distribution. Furthermore, forecasters are relatively in agreement about the mean predicted growth rate in good times, while in bad times there is large disagreement among forecasters about the mean predicted growth 

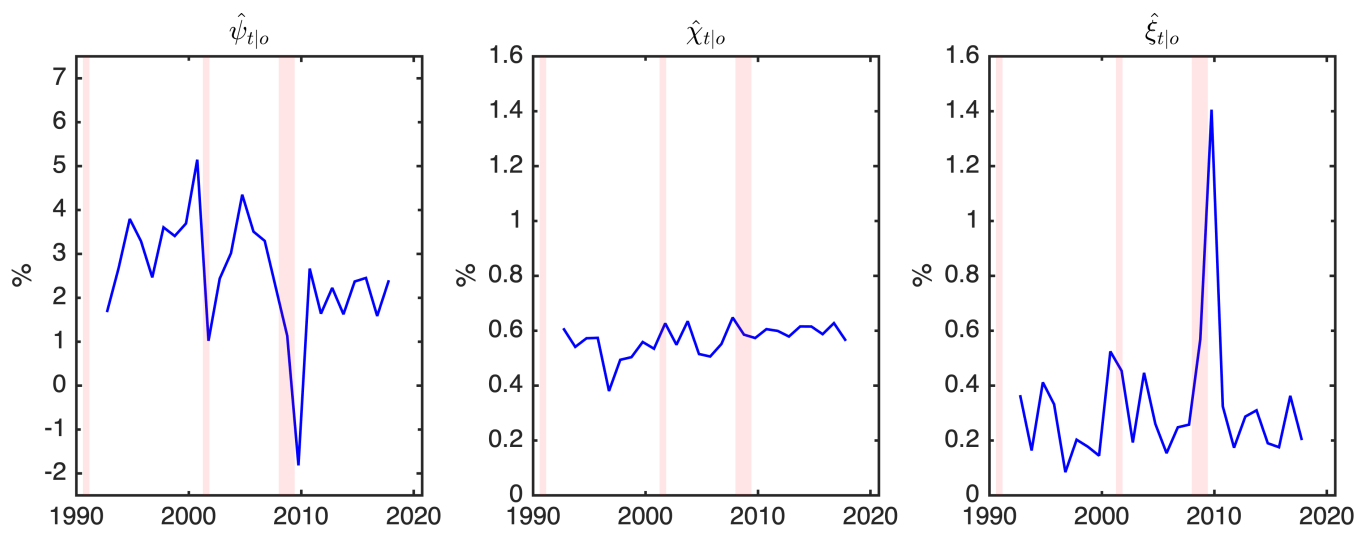

Figure 5.1: Plot of the moments of the densities extracted from SPF survey responses when asked to predict a probability distribution for the real GDP growth rate. $\hat{\psi}_{t \mid o}$ represents the average forecasted growth rate, $\hat{\chi}_{t \mid o}$ represents dispersion within a forecasters forecast, and $\hat{\xi}_{t \mid o}$ represents dispersion across forecasters. Plot generated using $o=4$. Red highlights shows NBER recession periods. The plotted date is the date when the forecast is made. All values are annualized and in percent.

rate.

In Appendix B, I do various robustness checks on the properties of uncertainty and disagreement. I compute another proxy of uncertainty by plotting the fraction of respondents who answered "Uncertain" or "Don't know" when asked to predict business conditions in the Michigan Survey of Consumers. I compute another proxy of disagreement by plotting the standard deviation across participants in forecasts of the S\&P500 earnings from Thompson Reuters IBES (Institutional Broker's Estimate System) Data. In addition, I also analyze the role of the 2008 financial crisis on my results. 


\section{CHAPTER 6 CALIBRATION}

\subsection{Macro Parameters}

To calibrate the model, I use relatively standard macro-parameters as shown in Table 6.1. Due to the availability of density forecasts being annual-average of annual-average growth rates, I set the model to have annual time periods, I use a discount rate of .95 and risk aversion of 1. By using log utility, I avoid the potential problems introduced by the separability of consumption and labor illustrated in [17]. I set the elasticity of labor supply to 1.5 , and set the capital share of production to .3 with a depreciation of $10 \%$. I calibrate the evolution of productivity using the utilization-adjusted TFP series from [10]. Since my model is stationary, I remove the linear trend and fit the resulting series to an AR(1) process. This estimation leads to a persistence of .91 and a annual standard deviation of 5.14\%.

In my model, I rule out stochastic volatility in the evolution of productivity. To check this assumption is reasonable, I check the partial autocorrelation function of the squared errors from the $\mathrm{AR}(1)$ regression. I find that the coefficients are small (around .2) and not significant. As a result, I rule out stochastic volatility in productivity. However, I note that the volatility of the shock to productivity drives a large fraction of the variance in aggregate variables. Uncertainty and disagreement, while statistically significant in explaining movements in macrovariables, make up a small portion of the total variance. I discuss this effect more in Chapter 7.

\subsection{Uncertainty and Disagreement}

To fit uncertainty and disagreement, I fit an $\mathrm{AR}(1)$ process to the empirical time-series setting $a_{t} \approx \hat{\chi}_{t \mid o}, b_{t} \approx \hat{\xi}_{t \mid o}$. I find the values in Table 6.2. 


\begin{tabular}{|c|c|c|}
\hline Parameter & Role & Value \\
\hline$\beta$ & Discount Rate & .95 \\
$\gamma$ & Risk Aversion & 1 \\
$\nu$ & Inverse Elasticity of Labor Supply & 1.5 \\
$\alpha$ & Capital Share in Production & .3 \\
$\delta$ & Depreciation Rate & .1 \\
\hline$\rho_{s}$ & Persistence of TFP & .91 \\
$\sigma_{s}$ & Stdev. of TFP & $5.14 \%$ \\
\hline
\end{tabular}

Table 6.1: Baseline parameter values. Model is calibrated for annual data. The evolution of TFP fit to detrended series from [10].

I find the steady state values of $\bar{a}, \bar{b}$ to be $.56 \%$ and $.32 \%$. Thus on average, I find that the SPF data implies the dispersion within each agent's individual beliefs about productivity is higher than the dispersion across agents. I find the persistence of uncertainty and disagreement are .25 and .19 respectively. Consistent with the time-series trends of $\chi_{t}, \xi_{t}$, the evolution of uncertainty has a smaller standard deviation when compared to the evolution of disagreement. I find in my $\mathrm{AR}(1)$ specification, that uncertainty has a standard deviation of $.06 \%$ whereas disagreement is $.25 \%$ reflecting the large change in dispersion across agents during recession periods. In addition, I observe a correlation of .22 for between $\epsilon_{t+1}^{a}$ and $\epsilon_{t+1}^{b}$. To quantify this magnitude, I simulate the correlation between two vectors of length 26, corresponding to the annual data from 1992-2017, using independent random normal draws. I find that .22 represents only 1.1 standard deviations away from the mean. I take this to be evidence that my assumption that the shocks to uncertainty and disagreement are not unreasonable.

\subsection{Fears of Model Misspecification}

To calibrate $\theta$, I first use US macrodata to see in reduced form what impact, if any, uncertainty and disagreement have on aggregate variables. Since my model is stationary, I 


\begin{tabular}{|c|c|c|}
\hline Parameter & Role & Value \\
\hline $\bar{a}$ & Stry Mean of Uncertainty & $.56 \%$ \\
$\rho_{a}$ & Persistence of Uncertainty & .25 \\
$\sigma_{a}$ & Stdev. of Uncertainty & $.06 \%$ \\
\hline $\bar{b}$ & Stry Mean of Disagreement & $.32 \%$ \\
$\rho_{b}$ & Persistence of Disagreement & .19 \\
$\sigma_{b}$ & Stdev. of Disagreement & $.25 \%$ \\
\hline
\end{tabular}

Table 6.2: Uncertainty and disagreement parameter values. Values fit to data from SPF calibrated to annual data setting $o=4$.

\begin{tabular}{r|rrrr}
\hline \hline & Hours Worked & Consumption & Investment & Output \\
\hline Uncertainty & $-58.73^{* *}$ & $-36.53^{* *}$ & $-93.54^{* *}$ & $-47.27^{* *}$ \\
& $(23.44)$ & $(16.66)$ & $(41.16)$ & $(19.68)$ \\
Disagreement & -3.71 & -0.13 & $-18.37^{*}$ & -1.97 \\
& $(5.2)$ & $(3.76)$ & $(10.16)$ & $(4.43)$ \\
TFP & 0.07 & $0.3^{* *}$ & $0.57^{* *}$ & $0.29^{* *}$ \\
& $(11.83)$ & $(10.51)$ & $(18.69)$ & $(11.88)$ \\
\hline$R^{2}$ & 0.25 & 0.42 & 0.37 & 0.37 \\
$\mathrm{~N}$ & 26 & 26 & 26 & 26 \\
\hline \hline
\end{tabular}

Table 6.3: Effects of uncertainty and disagreement on US aggregate variables. Aggregate variables represented as percent deviations from steady state. Productivity, uncertainty, and disagreement represented in percent. Newey-West robust standard errors shown.

\begin{tabular}{r|rrrr|rrrr}
\hline \hline & \multicolumn{5}{|c|}{ Data } & \multicolumn{5}{c}{ Model } \\
& Hours & Cons. & Inv. & Output & Hours & Cons. & Inv. & Output \\
\hline Uncertainty & -58.73 & -36.53 & -93.54 & -47.27 & -52.46 & -44.03 & -26.92 & -40.58 \\
& $(23.44)$ & $(16.66)$ & $(41.16)$ & $(19.68)$ & & & & \\
Disagreement & -3.71 & -0.13 & -18.37 & -1.97 & -11.20 & -2.84 & -28.33 & -7.98 \\
& $(5.2)$ & $(3.76)$ & $(10.16)$ & $(4.43)$ & & & & \\
\hline \hline
\end{tabular}

Table 6.4: Data and model predicted effects of uncertainty and disagreement on aggregate variables. $\theta$ is calibrated to fit an evenly weighted sum of the effect of uncertainty and disagreement on labor and consumption. Aggregate variables represented as percent deviations from steady state. Productivity, uncertainty, and disagreement represented in percent. Newey-West standard errors from the regression shown in parenthesis. 
remove the linear trend from the deflated series and compute percent deviations from the mean for US hours worked, consumption, output, and investment. ${ }^{1}$ I then regress these aggregate variables on uncertainty and disagreement controlling for changes in TFP using the detrended series from [10]. I use the specification

$$
Y_{t}=\beta_{0}+\beta_{1}(\text { Uncertainty })_{t}+\beta_{2}(\text { Disagreement })_{t}+\beta_{3}(\mathrm{TFP})_{t}+\epsilon_{t}
$$

where $Y_{t}$ represents various aggregate variables. I show the results of my regression in Table 6.3.

To calibrate $\theta$, I match an equal weighted sum of the effects of uncertainty and disagreement on the agent's controls of labor and consumption. ${ }^{2}$ I find that setting $\theta=30$ gives me the optimal fit between the model and reduced form effects of uncertainty and disagreement. I show the calibrated results in Table 6.4.

1. I detail the sources I use to construct US aggregate data in Appendix A.

2. The results are largely unchanged if I instead fit an equal weighted sum of the effects of uncertainty and disagreement on all four aggregate variables. 


\section{CHAPTER 7 \\ RESULTS}

\subsection{Uncertainty and Disagreement on Aggregate Variables}

I find that my defined concepts of uncertainty and disagreement have statistically significant empirical impact on aggregate variables in reduced form. While uncertainty has large impacts on hours worked, consumption, investment, and output, I find disagreement has a large significant effect on investment. The coefficients for disagreement on hours worked, consumption, and output are smaller and not statistically significant. I find an increase in the dispersion within each forecaster's subjective distribution and an increase in the dispersion across forecaster's in the predicted mean growth rate both correlates with a decrease in aggregate variables with the effect of uncertainty being larger than that of disagreement.

Looking at the results of Table 6.4, I find that the model can match the coefficients for the effects of uncertainty and disagreement on aggregate variables reasonably well. While investment dynamics remain a challenge due to the lack of adjustment costs or sticky prices, the model can match the effects of uncertainty and disagreement on aggregate variables within one standard deviation of the reduced form coefficients.

The fact that my model is able to match both the magnitude and the sign of the effects of uncertainty and disagreement on aggregate variables is not trivial. In the model, the effect of $a_{t}, b_{t}$ on the controls comes through the mean distortion from fears of model misspecification. As shown in Equation (3.20), the mean distortion is proportional to the parameter $\theta$. Thus the calibration of $\theta$ while amplifying the total magnitude of the effects of uncertainty and disagreement do not affect their relative magnitudes or signs. As a result, the fit I find in Table 6.4 shows evidence that my model mechanism can explain the data.

To understand the mechanism behind my results, first consider an increase in uncertainty. Each agent then has greater dispersion in his first order belief about productivity. In my 
model, this effect corresponds to lower signal accuracy and a higher variance for the posterior distribution of $s_{t}$. Since each agent worries about the distribution of $s_{t}$ being potentially misspecified, the agent makes a cautious adjustment in his forecast of $s_{t}$. Given the increased variance, the resulting negative mean shift is larger, and the expected productivity under the distorted measure is lower. The agent then chooses labor and consumption under the distortion which has a similar effect to a negative TFP shock. The increase in uncertainty lowers both his marginal product of labor and marginal utility. As a result, he adjusts both his labor and consumption downward. In my model, all agents ex-post behave the same, and thus aggregate labor and consumption are both adjusted downward and in turn affect investment and output.

When disagreement increases, each agent believes there is greater dispersion in the predicted productivity across agents. This effect corresponds to lower correlation in the signals across agents. Note that $b_{t}$ does not affect the posterior distribution for $s_{t}$. Thus the effect of dispersion across agents enters through higher order beliefs via the posterior for $\zeta_{t}$. Under fears for model misspecification, the agent makes a cautious adjustment toward the case where they expect a lower intermediate goods price and a lower marginal product of labor and chooses to adjust labor, output, consumption, and investment downward

The difference in magnitude arises in my model since productivity is the key variable which agents care about. Under fears of model misspecification, an increase in uncertainty causes agents to believe productivity will be low. On the other hand, an increase in disagreement affects the terms of trade and does not affect an individual agent's view about productivity. As a result, uncertainty and first order beliefs have a larger effects on total goods production since it enters the agent's problem directly rather than disagreement and higher order beliefs which would enter through the intermediate goods price. 

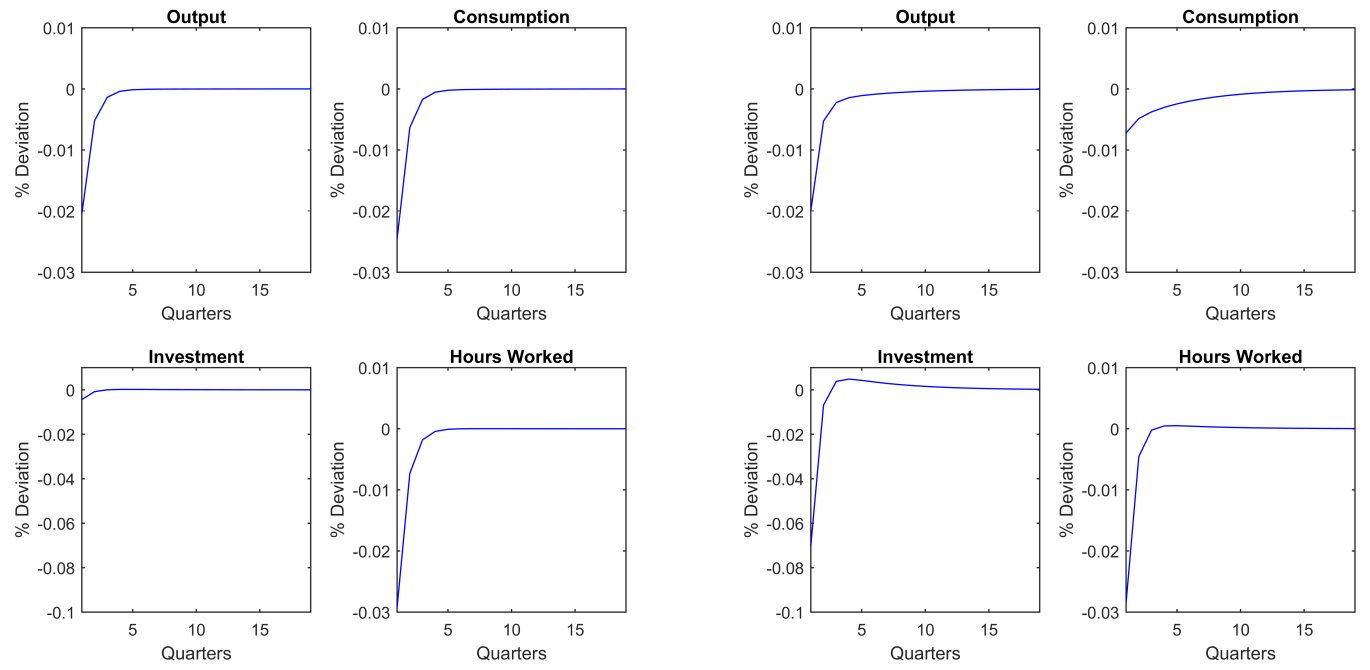

Figure 7.1: Model predicted response of aggregate variables to a one time one standard deviation shock to uncertainty and disagreement. Uncertainty shown in the left panel and disagreement shown in the right panel. All variables shown as percent deviations from steady state.

\subsection{Impulse Response Functions}

To see the effects of the changing distribution of signals on the macrovariables scaled by the shock variance. I plot the impulse response function to a one standard deviation shock in uncertainty and disagreement for the equal-weighted calibration of $\theta$ in Figure 7.1.

Looking at the impulse response functions, I note that uncertainty and disagreement have similar importance when scaled by the volatility of the respective shocks. Thus while uncertainty matters more for the agent, it has less volatility in its time-series. In contrast, disagreement matters less for each agent and varies more. For output, I find that both uncertainty and disagreement have a similar effect, Interestingly, uncertainty has larger effects on consumption, while disagreement has larger effects on investment. 

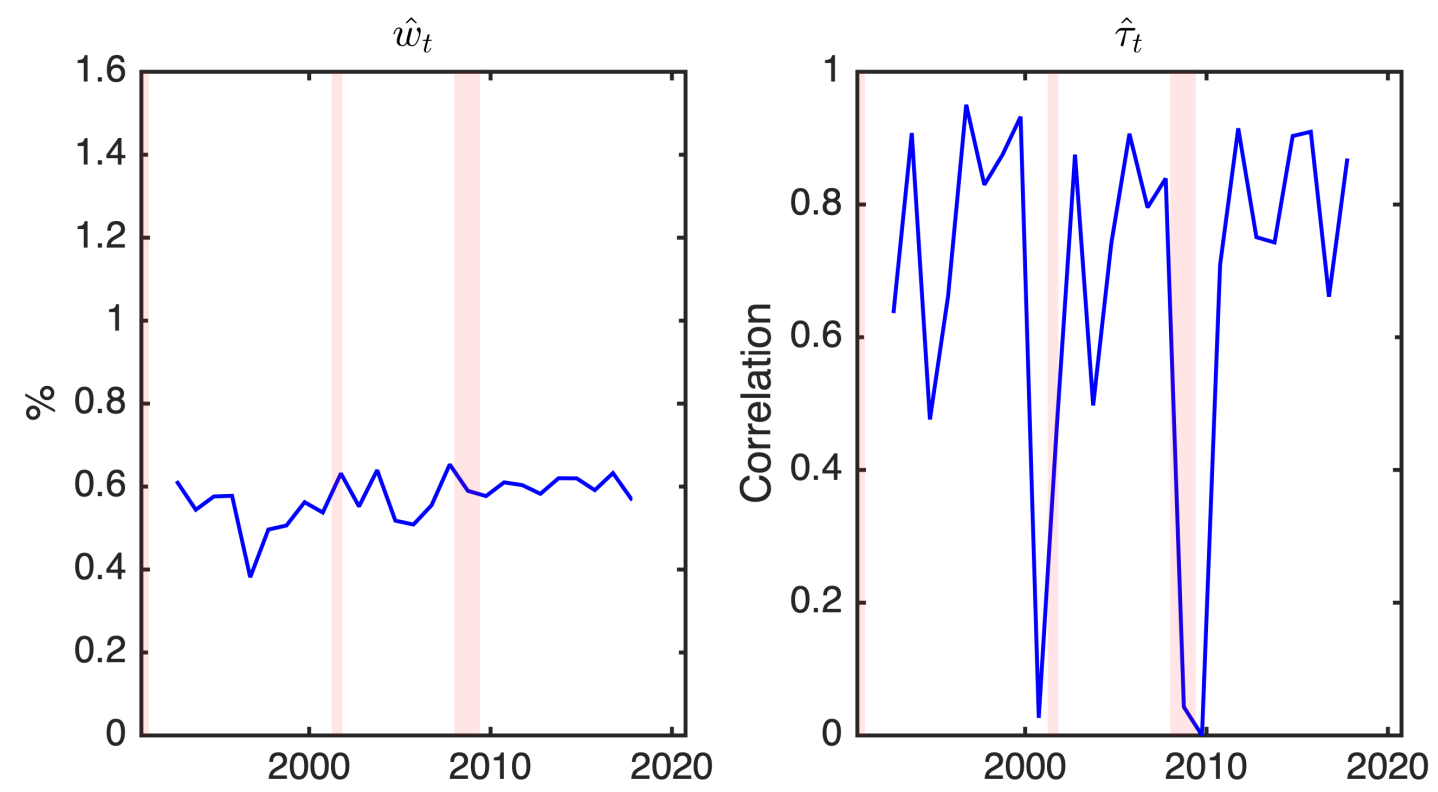

Figure 7.2: Implied time series for the signal volatility $\left(w_{t}\right)$ and correlation $\left(\tau_{t}\right)$ using uncertainty and disagreement fit to SPF data setting $o=4$. Red highlights show NBER recession periods. Plotted date is when forecast is made. Values are for annual time periods.

\subsection{Distribution of Signals}

Using the time-series for uncertainty and disagreement, I plot the implied time-series for the volatility of the signal $w_{t}$ and the correlation of signal across agents $\tau_{t}$ in Figure 7.2.

I find that my structural model implies that signal accuracy remains relatively fixed over time around $.5 \%$. This result is consistent with the time-series properties of uncertainty, since they are directly related. I find that the time-series of the correlation of signals across agents varies widely. Notably, the correlation seems to hit the bound of 0 for at least two consecutive periods during the recession bands within my sample. While my sample is limited due to the availability of SPF data, this evidence is suggestive that during recessions there are periods of minimal correlation in the signal across agents combined with low signal accuracy (high $w_{t}$ ). 


\subsection{Magnitude of Probability Distortions}

Given my calibrated value of $\theta$ for my model, I analyze how realistic the worst case model chosen by the agent is for $s_{t}, \zeta_{t}$. Since under the data generating process, I assumed each agent ends up receiving the same signal, I focus on the mean distortion to productivity $s_{t}$. For convenience, I analyze the distortion to the shock $\epsilon_{i, t}^{z}$ which is the first entry of the vector $w_{i, t}^{1}$. I find that my model implies

$$
\begin{aligned}
& \epsilon_{i, t}^{z} \sim \begin{cases}\mathcal{N}\left(0, a_{t}^{2}\right) & \text { Undistorted } \\
\mathcal{N}\left(-v_{t}, a_{t}^{2}\right) & \text { Distorted }\end{cases} \\
& v_{t}=-\left[\begin{array}{ll}
1 & 0
\end{array}\right] \theta V_{x^{2}}^{2} X_{w^{1}} \Sigma_{t}^{s, \zeta}
\end{aligned}
$$

To quantify the statistical distance between the undistorted and distorted measure, I consider testing the null hypothesis that draws of $\epsilon_{i, t}^{z}$ come from a normal with the distorted mean against the alternative that they come from a normal distribution with a different mean.

Let $\hat{\epsilon}_{1}, \hat{\epsilon}_{2}, \ldots, \hat{\epsilon}_{n}$ denote IID draws from the distribution of $\epsilon_{i, t}^{z}$. As an approximation, I fix $a_{t}, b_{t}$ at their steady state values. I can then derive the distribution of the sample mean $S_{n}=\frac{1}{n} \sum_{i=1}^{n} \hat{\epsilon}_{i}$ under the null hypothesis

$$
\frac{\sqrt{n}\left(S_{n}+\bar{v}\right)}{\bar{a}} \sim \mathcal{N}(0,1)
$$

Since I assumed the undistorted specification for $s_{t} \mid z_{i, t}$ is the data generating process, I set $S_{n}=0$, since $S_{n}$ has expectation 0 under the undistorted model. I then solve for the number of data points required to reject the null hypothesis with $95 \%$ confidence.

I find $n \geq 1.5$ for my even-weighted calibration. Under my calibrated level of fears of model misspecifcation, it would take 1.5 years for an agent in my model to reject that his 
worst case model is not the correct specification with $95 \%$ confidence. I find this to be a reasonable level of concern for model ambiguity since business cycles are typically between 2-8 years. Thus within the time scale pertinent to business cycles, I find that my agent is unable to fully reject his worst case model as representing the correct dynamics for the system.

\subsection{Aggregate Moments}

I find that my results differ from the findings of [1] who also analyze the movement of higher order beliefs and their impact of aggregate variables. To provide a comparison to their results, I compute aggregate moments for US aggregate data using a comparable method to their paper. Writing variables as percent deviation from steady state, I apply the bandpass filter from [6] at the 2-8 year frequencies. I compute moments for three experiments. In the first experiment, I generate moments for my original calibration from Chapter 6 setting $\theta=0$ which shuts down any variation in controls due to dynamics in uncertainty and disagreement. In the second experiment, I generate moments from my original calibration from Chapter 6 setting $\theta=30$ which matches the calibrated value. In the third experiment, following the calibration strategy of [1], I calibrate $\sigma_{s}, \sigma_{a}, \sigma_{b}$ holding $\theta=30$ and all other parameters fixed to match an equal weighted sum of the moments for the standard deviation of output, labor, consumption and investment. To provide a reference to the actual data moments, I also compute aggregate moments using US aggregate data from 1960 to 2017. I show the results of the experiments in Table 7.1.

Looking at the aggregate moments for the first and second experiment, I find that the model does not match the volatility of US aggregate data well. Comparing the first and second experiments, I find that the variation in uncertainty and disagreement calibrated from SPF data makes a negligible differences for aggregate moments. This result implies volatility in TFP drives aggregate moments. In line with RBC models where TFP is stochastic, I find 


\begin{tabular}{l|c|c|c|c|c}
\hline \hline & Data & From [1] & Exper. 1 & Exper. 2 & Exper. 3 \\
\hline $\operatorname{stdev}(\mathrm{y})$ & 1.30 & 1.52 & 3.85 & 3.85 & 2.77 \\
$\operatorname{stdev}(\mathrm{n})$ & 1.60 & 1.52 & 0.39 & 0.39 & 0.50 \\
$\operatorname{stdev}(\mathrm{c})$ & 0.76 & 0.69 & 0.84 & 0.83 & 0.70 \\
$\operatorname{stdev}(\mathrm{i})$ & 5.01 & 5.00 & 6.15 & 6.05 & 4.22 \\
$\operatorname{stdev}(\mathrm{y} / \mathrm{n})$ & 0.75 & 0.91 & 3.10 & 3.10 & 2.20 \\
\hline $\operatorname{corr}(\mathrm{c}, \mathrm{y})$ & 0.87 & 0.89 & 0.97 & 0.97 & 0.95 \\
$\operatorname{corr}(\mathrm{i}, \mathrm{y})$ & 0.95 & 0.97 & 0.99 & 0.99 & 0.98 \\
$\operatorname{corr}(\mathrm{n}, \mathrm{y})$ & 0.89 & 0.82 & 0.96 & 0.96 & 0.75 \\
$\operatorname{corr}(\mathrm{c}, \mathrm{n})$ & 0.88 & 0.49 & 0.93 & 0.92 & 0.85 \\
$\operatorname{corr}(\mathrm{i}, \mathrm{n})$ & 0.88 & 0.92 & 0.96 & 0.96 & 0.64 \\
$\operatorname{corr}(\mathrm{c}, \mathrm{i})$ & 0.81 & 0.76 & 0.92 & 0.92 & 0.87 \\
\hline $\operatorname{corr}(\mathrm{y}, \mathrm{y} / \mathrm{n})$ & -0.15 & 0.29 & 1.00 & 1.00 & 0.96 \\
$\operatorname{corr}(\mathrm{n}, \mathrm{y} / \mathrm{n})$ & -0.59 & -0.30 & 1.00 & 1.00 & 0.96 \\
\hline \hline
\end{tabular}

Table 7.1: Data and model simulated aggregate moments. Moments represented as percent deviation from steady state. US aggregate data shown in the left column. Results from three experiments and comparison with results from [1] shown.

I can match the correlations between macrovariables reasonably well. However, the model implies a very high correlation between labor productivity and output a problem common to single shock RBC models. Looking at the results for the third experiment, I find that although the freedom to choose $\sigma_{s}, \sigma_{a}, \sigma_{b}$ allows me to match the standard deviations of macrovariables better while maintaining a good fit for the correlations, it does not help address the high correlations between labor productivity and output.

Overall, I find that my model mechanism is unable to generate the success of [1] in matching the low correlation of labor productivity with output. However, in their paper they do not directly discipline movements in higher order beliefs and instead freely choose the evolution of these beliefs to match aggregate moments to data. My inability to generate their results suggests that uncertainty and disagreement are unlikely to be the main underlying mechanism which drives higher order beliefs in their model. In addition, I find given the structure and restrictions from the signal structure in my model, no amount of variation in uncertainty and disagreement can account for the large effects on the labor productivity 
correlations noted in their results.

While my model mechanism represents only a small portion of the total variation in aggregate variables, the mechanism captures movements in beliefs coming from changes in two properties of the distribution of signals. I do not allow other sources such as ex-ante heterogeneity or different information sets across agents. Thus while I am unable to justify the large deviations in higher order beliefs used in their paper to generate their moments, I find my results motivate the study of alternative explanations that could lead to large shifts in the higher order beliefs. 


\section{CHAPTER 8}

\section{DISCUSSION AND EXTENSIONS}

The results of my model show that the effect of signals on an agent's decision is an important mechanism to be studied further. The implications of my model suggest more research is needed where a density rather than just a single number is used to understand agents' expectations, a viewpoint echoed in [13]. The SPF is one of the few surveys which ask forecasters for density estimates. I note the existing literature uses dispersion across forecasters to measure deviations in first order beliefs. Such a method could potentially bias results as well as having potentially incorrect implications.

In addition, my paper shows how additional structure can be used along with dispersion across forecasters to quantify the role of higher order beliefs. This methodology augments existing work in the literature to measure of the role of higher order beliefs directly using surveys such as [8]. While, my model restricts only first and second order beliefs from entering in the agent's problem through the first order perturbation, my results motivate further study into quantifying how many order of beliefs and the effect of these beliefs on economic agents.

The aggregate effects in my model also motivate further research about how agents extract a signal from a variety of different sources of information. My model assumes a tractable structure in the distribution of signals. By understanding how different agents process information into a private signal, one could potentially look to measure the qualitative properties of information directly from the sources rather than using implied measures from forecasts of macroeconomic outcomes.

In my model, fears of model misspecification drive agents to adjust their first and higher order beliefs when the distribution of signals changes. Given my model is compatible with robustness, one could potentially consider a case where agents have different models for the evolution of productivity rather than signals. The addition of potential model ambiguity 
introduces changes in the dynamics of productivity and could have time-varying effects which could be interesting to study.

The results of my model are subject to two key caveats. First, my model assumes that agents provide a normally distributed forecast for the growth rate of real GDP. I find that the distributions in the survey data often tend to exhibit skew and kurtosis inconsistent with a normal distribution. I expect the model predictions will be sensitive to distributional assumptions especially with respect to weight in the left tail. Second, I restrict the signal accuracy and correlation structure to be homogeneous across agents in my model. While this assumption allows me to tractably solve the model, it imposes restrictions on the dynamics of uncertainty and disagreement. I do not find that these restrictions are violated in the available sample of SPF data, however, a more general signal structure may potentially affect the amount of variation in aggregate variables that uncertainty and disagreement capture. I leave these two points as potentially fruitful areas of future extension. 


\section{CHAPTER 9 CONCLUSION}

In this paper, I present a model to understand how changes in the distribution of signals about unknown economic conditions affect an agent's controls in a business cycle model. I analyze how the changes in signal distribution relate to dispersion both within and across agents' forecasts about future conditions and how these movements affect their choices in a dynamic stochastic general equilibrium model with incomplete information and fears of model misspecification.

I define two key concepts, uncertainty and disagreement, which capture changes in information sources available to agents and can be linked to data. I define uncertainty as the dispersion in each agent's forecast about economic conditions and disagreement as the dispersion across agents in their mean forecasts about economic conditions. I show that uncertainty affects agent's controls through first order beliefs and disagreement through higher order beliefs.

Using data from the SPF, I extract empirical counterparts for uncertainty and disagreement. I find that uncertainty stays relatively constant over the business cycle while there is evidence to suggest disagreement spikes during recession periods. I find uncertainty and disagreement have statistically significant effects on US aggregate macrovariables. Calibrating the model, I find my model mechanism can parsimoniously match the sign and magnitude of these predicted effects suggesting the mechanism is relevant for business cycle dynamics. However, I find evidence that dynamics in the signal accuracy and correlation structure do not capture a significant portion of variation in aggregate variables in contrast to [1].

My model provides insight into the formulation of first and higher order beliefs about unknown economic conditions and show they have important consequences for agents' decision making throughout the business cycle. I illustrate how to capture movements in beliefs using uncertainty and disagreement which can be linked to data. The results of my model 
suggest potential avenues of further research to better understand how beliefs are formulated and other sources of dynamics that are important for explaining the behavior of agents in the macroeconomy. 


\section{REFERENCES}

[1] George-Marios Angeletos, Fabrice Collard, and Harris Dellas. Quantifying confidence. Econometrica, 86(5):1689-1726, September 2018.

[2] George-Marios Angeletos and Jennifer La'O. Sentiments. Econometrica, 81(2):739-779, 2013.

[3] Anmol Bhandari, Jaroslav Borovicka, and Paul Ho. Survey data and subjective beliefs in business cycle models. Working Paper, November 2017.

[4] Pedro Bordalo, Nicola Gennaioli, Yueran Ma, and Andrei Schleifer. Over-reaction in macroeconomic expectations. Working Paper, December 2017.

[5] Jaroslav Borovicka and Lars P. Hansen. Notes on second order approximations of markov solutions, February 2018.

[6] Lawrence J. Christiano and Terry J. Fitzgerald. The band pass filter. International Economic Review, 44(2):435-465, 2003.

[7] Olivier Coibion and Yuriy Gorodnichenko. Information rigidity and the expectations formation process: A simple framework and new facts. American Economic Review, 105(8):2644-2678, August 2015.

[8] Olivier Coibion, Yuriy Gorodnichenko, Saten Kumar, and Jane Ryngaert. Do you know that i know that you know...? higher-order beliefs in survey data. NBER Working Paper, September 2018.

[9] Joseph Engelberg, Charles F. Manski, and Jared Wiliams. Comparing the point predictions and subjective probability distributions of professional forecasters. Journal of Business and Economic Statistics, 27(1):30-41, 2009.

[10] John Fernald. A quarterly, utilization-adjusted series on total factor productivity. Working Paper, April 2014.

[11] Lars P. Hansen and Thomas J. Sargent. Robust control and model uncertainty. American Economic Review, 91(2):60-66, 2001.

[12] Zhen Huo and Naoki Takayama. Higher order beliefs, confidence, and business cycles. Working Paper, January 2015.

[13] Charles F. Manski. Survey Measurement of Probabilistic Macroeconomic Expectations: Progress and Promise, chapter 5, pages 411-471. University of Chicago Press, 2018.

[14] Kristoffer P. Nimark. Dynamic higher order expectations. Working Paper, March 2017.

[15] Stephanie Schmitt-Grohe and Martin Uribe. Solving dynamic general equilibrium models using a second-order approximation to the policy function. Journal of Economic Dynamics and Control, 28:755-775, 2004. 
[16] Robert M. Townsend. Forecasting the forecasts of others. Journal of Political Economy, 91(4):546-588, 1983.

[17] Mathias Trabandt and Harald Uhlig. The laffer curve revisited. Journal of Monetary Economics, 58:305-327, 2011. 


\section{APPENDIX A}

\section{APPENDIX: US AGGREGATE DATA SOURCES}

I detail the sources and construction of US aggregate data in Table A.1.

\begin{tabular}{cc}
\hline \hline Data & Formula \\
\hline Output & $\mathrm{Y}=\mathrm{GDP} / \mathrm{GDPDEF})$ \\
Consumption & $\mathrm{C}=(\mathrm{CND}+\mathrm{CS}) /(\mathrm{GDPDEF})$ \\
Investment & $\mathrm{I}=(\mathrm{CD}+\mathrm{FPI}+\mathrm{DI}) /(\mathrm{GDPDEF})$ \\
Hours Worked & $\mathrm{N}=(\mathrm{HOANBS})$ \\
TFP & $\mathrm{s}=\log (\mathrm{UTILTFP})$ \\
\hline Shorthand & Source \\
\hline GDP & http://research.stlouisfed.org/fred2/series/GDP \\
CND & http://research.stlouisfed.org/fred2/series/PCND \\
CD & http://research.stlouisfed.org/fred2/series/PCEDG \\
CS & http://research.stlouisfed.org/fred2/series/PCESV \\
FPI & http://research.stlouisfed.org/fred2/series/FPI \\
DI & http://research.stlouisfed.org/fred2/series/CBI \\
HOANBS & http://research.stlouisfed.org/fred2/series/HOANBS \\
GDPDEF & http://research.stlouisfed.org/fred2/series/GDPDEF \\
UTILTFP & https://www.frbsf.org/economic-research/files/quarterly_tfp.xlsx \\
\hline \hline
\end{tabular}

Table A.1: Data Sources for US Macrodata.

The data is from the St. Louis Federal Reserve Economic Database (FRED). All quantities are deflated using the implicit GDP deflator (GDPDEF). The series for productivity is the utilization based series from [10]. Since my model is stationary, I remove the linear trend in each resulting series and measure them as percent deviations from mean to match the first order perturbation used in my solution method. 


\section{APPENDIX B}

\section{APPENDIX: EXTRACTING UNCERTAINTY AND DISAGREEMENT}

\section{B.1 Time Period and Distributional Assumptions Within Bins}

In this section, I elaborate on the decision not to use the real GNP survey data prior to 1992 and elaborate on my assumption of a uniform distribution within bins. Given the signal structure of my model, I assume each agent predicts a normal distribution for the growth rate of real GDP. However, I find the density forecasts recorded in the SPF data often do not admit a nice normal fit. In this section, I analyze the effects on my extracted series and reduced form results. To examine this, I compare a uniform, normal, and generalized beta distributional fits to the data.

Using the same notation, I index participants $j \in\left\{1, \ldots, J_{t}\right\}$ where $J_{t}$ is the total number of participants at time $t$ and index bins $\omega \in\left\{1, \ldots \Omega_{t}\right\}$ where $\Omega_{t}$ is the total number of bins at time $t$. Let bin $\omega$ correspond to the interval $\left[u_{\omega}^{\ell}, u_{\omega}^{u}\right)$ and $\operatorname{Pr}_{j, t}(\omega)$ denote the response of participant $j$ at time $t$ to the probability that the growth rate lies in bin $\omega$.

Given the notation, I fit both a normal and a generalized beta distribution to the discrete data. To fit a normal distribution, I fit two parameters mean and variance. At each quarter $o$ in year $t$ for each participant $j$, I solve

$$
\min _{\mu_{t \mid o}^{j}, \sigma_{t \mid o}^{j}} \sum_{\omega=1}^{\Omega_{t}}\left\{\left[\Phi\left(u_{\omega}^{u}, \mu_{t \mid o}^{j}, \sigma_{t \mid o}^{j}\right)-\Phi\left(u_{\omega}^{\ell}, \mu_{t \mid o}^{j}, \sigma_{t \mid o}^{j}\right)\right]-\operatorname{Pr}_{t \mid o}^{j}(\omega)\right\}^{2}
$$

where $\Phi(u, \mu, \sigma)$ is the CDF of a normal with mean $\mu$ and variance $\sigma^{2}$ evaluated at $u$.

To fit a generalized beta distribution, I use a four parameter beta distribution with two shape parameters and two parameters governing the left and right endpoints. If the respondent uses bins that do not include the left most or right most bin, I restrict the fitted 

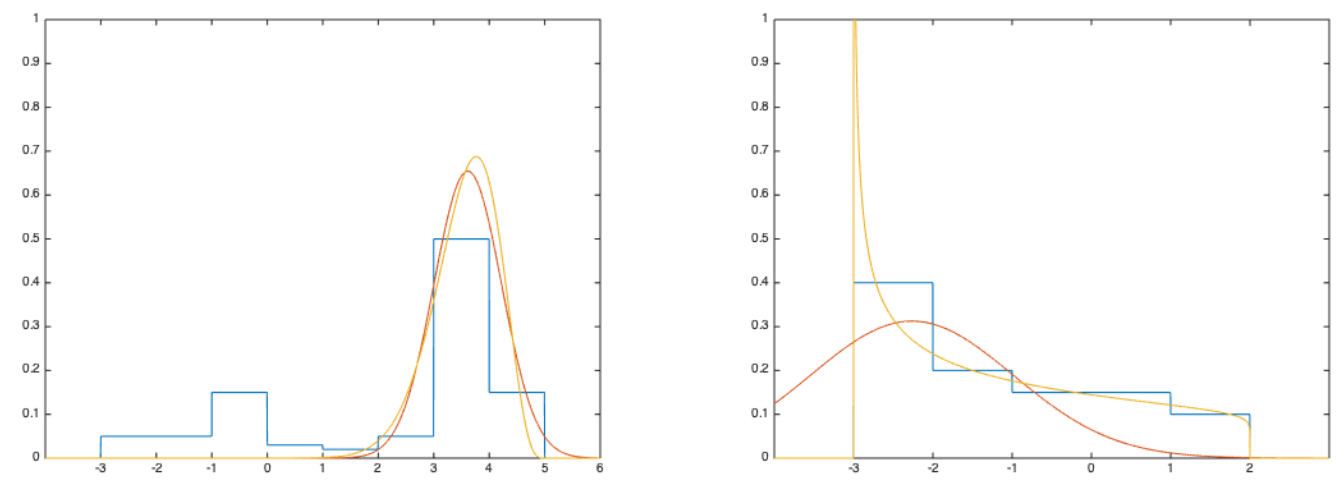

Figure B.1: Plot comparing PDFs for the uniform, normal, and beta distributional assumption for examples of responses in the SPF data. Blue shows the uniform fit results, red shows the normal fit results, and yellow shows the beta fit results. All values are annualized and in percent.

beta distribution to have the support of the range of responses. To mitigate the effect of truncation, if the survey respondent put non-zero probability in either of the left or right end bins, I set the end bins to the same width as the other bins. The use of the beta distribution is similar to the method used in [9]. The objective function then is given by

$$
\min _{\alpha_{t \mid o}^{j}, \beta_{t \mid o}^{j}} \sum_{\omega=1}^{\Omega_{t}}\left\{\left[\Psi\left(u_{\omega}^{u}, \alpha_{t \mid o}^{j}, \beta_{t \mid o}^{j}, \ell_{t \mid o}^{j}, r_{t \mid o}^{j}\right)-\Psi\left(u_{\omega}^{\ell}, \alpha_{t \mid o}^{j}, \beta_{t \mid o}^{j}, \ell_{t \mid o}^{j}, r_{t \mid o}^{j}\right)\right]-\operatorname{Pr}_{t \mid o}^{j}(\omega)\right\}^{2}
$$

where $\Psi(u, \alpha, \beta, \ell, r)$ represents the generalized beta distribution with parameters $\{\alpha, \beta, \ell, r\}$ evaluated at $u$.

I plot two example cases of potential issues with the parametric distributional assumptions in Figure B.1. In the left panel, the survey respondent provides a bimodal distribution, and in the right panel, the survey respondent provides an asymmetric distribution that is right-skewed. Note that respondent error is unlikely given the large number of bins used in the response. These two cases represent potential deviations between the data and my model assumptions. I find that in the left panel, both the normal and generalized beta are unable to fit the bimodal distribution. In the right panel, I find that the normal distribution 

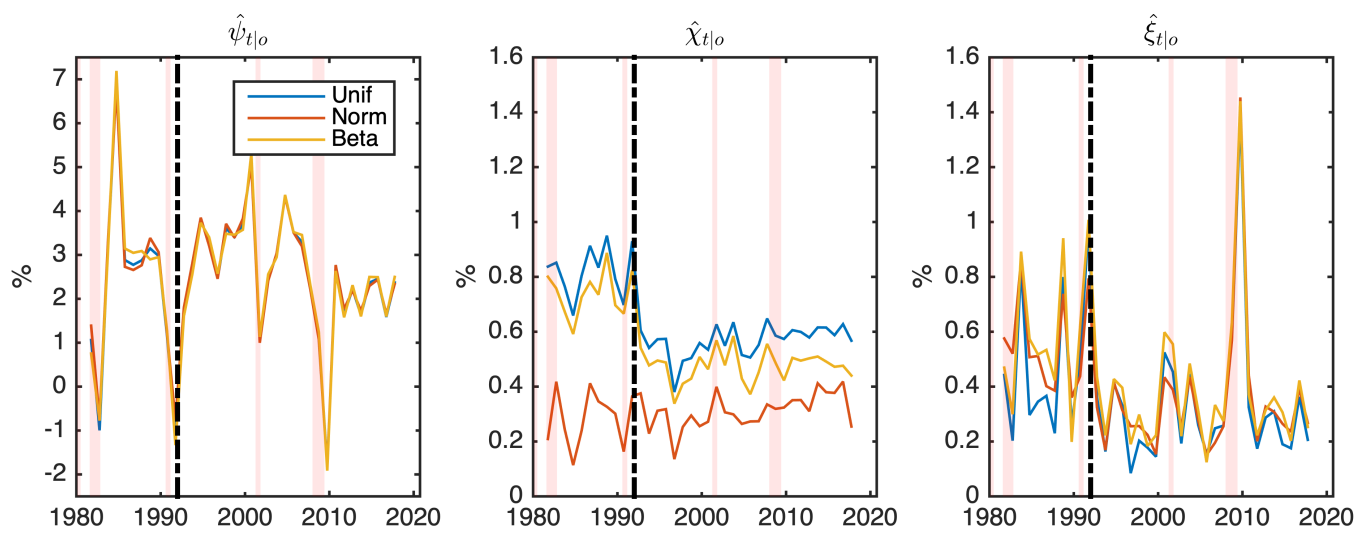

Figure B.2: Plot comparing estimates of $\hat{\psi}_{\left.t\right|_{o}}, \hat{\chi}_{t \mid o}$, and $\hat{\xi}_{\left.t\right|_{o}}$ using the uniform, normal, and generalized beta distribution fit for $o=4$. Blue shows the uniform fit results, red shows the normal fit results, and yellow shows the beta fit results. Red highlights show NBER recession periods. All values are annualized and in percent.

cannot fit the skew.

To compare the various parametric assumptions on the PDF of survey respondents, I compute the three corresponding moments $\hat{\psi}_{t \mid o}, \hat{\chi}_{t \mid o}, \hat{\xi}_{\left.t\right|_{o}}$ from Chapter 5 using the uniform, normal, and beta fits in Figure B.2 for the time period of 1981-2017 which includes the real GNP survey time period.

Looking at Figure B.2, I note that all three distributional assumptions are remarkably similar for the plot for $\hat{\psi}_{t \mid o}$, representing the average across agents in the mean of the fitted densities. This evidence suggests all three of the distributional assumptions are not very different in estimation of the first moment from the survey data.

In the plot for $\hat{\chi}_{t \mid o}$, which represents the average across agents in the standard deviation in their fitted densities, there is a discontinuous break between the pre-1992 data and the post 1992 fits for both the uniform and beta fits. In addition, I find that the uniform and beta fits estimate a systematically higher standard deviation in the densities than the normal fit. Interestingly the gap between the distributions for $\hat{\chi}_{t_{\mid o}}$ shrinks if I limit the sample to respondents who use a larger number of bins in their responses. This evidence suggests that the normal distribution has trouble fitting the densities when respondents use fewer 


\begin{tabular}{rr|rrrr}
\hline \hline & & Hours Worked & Consumption & Investment & Output \\
\hline \multirow{4}{*}{ Uncertainty } & Unif & $-58.73^{* *}$ & $-36.53^{* *}$ & $-93.54^{* *}$ & $-47.27^{* *}$ \\
& & $(23.44)$ & $(16.66)$ & $(41.16)$ & $(19.68)$ \\
& \multirow{6}{*}{ Norm } & $-54.75^{* *}$ & $-37.48^{* *}$ & $-90.61^{* *}$ & $-45.99^{* *}$ \\
& & $(17.88)$ & $(12.5)$ & $(28.21)$ & $(14.24)$ \\
& \multirow{2}{*}{ Beta } & -15.82 & -4.92 & -29.18 & -9.31 \\
& & $(24.17)$ & $(15.1)$ & $(38.26)$ & $(18.57)$ \\
\hline \multirow{3}{*}{ Unisagreement } & -3.71 & -0.13 & $-18.37^{*}$ & -1.97 \\
& \multirow{2}{*}{ Norm } & $(5.2)$ & $(3.76)$ & $(10.16)$ & $(4.43)$ \\
& & $-6.7^{* *}$ & -1.86 & $-23.9^{* *}$ & $-4.22^{*}$ \\
& \multirow{2}{*}{ Beta } & $(2.48)$ & $(2.02)$ & $(4.75)$ & $(2.17)$ \\
& & -5.82 & -1.63 & $-21.6^{* *}$ & -3.84 \\
& & $(4.27)$ & $(2.58)$ & $(8.86)$ & $(3.24)$ \\
\hline \hline
\end{tabular}

Table B.1: Comparison of coefficients from the regression in equation (6.1) for varying distributional assumptions. Aggregate variables represented as percent deviations from steady state. Productivity, uncertainty, and disagreement represented in percent. Newey-West robust standard errors shown.

bins. The uniform and beta have more flexibility and are able to capture these cases better. In addition, due to the discontinuous break at 1992, I restrict the sample to be post-1992. Looking at the plot for $\hat{\xi}_{t \mid o}$, representing the volatility across agents in the fitted mean, I find qualitatively the results are similar across the three fits. I find that the uniform and beta fit have slightly more volatility which may be due to the fact that they are more flexible than the normal. However, both the uniform and beta distribution assume an underlying which is unimodal.

For the post-1992 data, I find that for uncertainty the series assuming a uniform has correlation .27 with the series assuming a normal and a correlation of .97 with the series assuming a beta. For disagreement, I find the series assuming a uniform has correlation .93 with the series assuming a normal and a correlation of .97 with the series assuming a beta.

To see the effects of the distributional assumptions on the reduced form effects of uncertainty and disagreement, I run the same regression in Equation (6.1) comparing coefficients across different distributions in Table B.1. 
I find that the reduced form results are relatively stable across the different distributional assumptions. I find that the regression coefficients which are statistically significant do not change in magnitude across the distributional assumptions. I also find the coefficients which are not statistically significant are within a two standard deviation interval for the statistically significant coefficients. Thus, while my model's assumption of a normal distribution is not always consistent with the SPF data, I find the quantitative effects are not substantially large.

In addition, I calibrate the $\mathrm{AR}(1)$ evolution of $a_{t}, b_{t}$ using the normal and beta distributional assumptions. For both the normal and beta cases, I find the steady state value of $\bar{b}$ violates the upper bound on $b_{t}$ imposed by the structure of my model. As a result, I use the uniform distribution which poses a nice balance between flexibility in the distribution as well as capturing the relevant moments of density forecasts from survey respondents. The

violation of the upper bound implies that the covariance matrix $\Sigma_{t}^{s, \zeta}$ is no longer positive semi-definite. However, setting this issue aside, I can still compute results for my model using the calibrated values for $a_{t}, b_{t}$. Holding the macro parameters in Table 6.1 fixed, I repeat the same exercise to calibrate $\theta$. I show the comparison of the data and model for the normal and beta distributions in Table B.2.

I find that the ability of my model to fit the effects of uncertainty and disagreement on aggregate variables is largely unaffected by the distributional changes. I find that with the exception of the effect of uncertainty on investment, the model coefficients are within a one standard deviation range of the regression coefficients consistent with the results under assuming the distribution within bins is uniform.

\section{B.2 Evidence against the Effect of Binning}

To ensure that the discrete binning is not a significant factor in the fitting of a normal distribution in the extraction of uncertainty, I use data from the European Central Bank 


\begin{tabular}{rr|rrrr|rrrr}
\hline \hline & & \multicolumn{6}{|c|}{ Data } & \multicolumn{4}{c}{ Model } \\
& & Hours & Cons. & Inv. & Output & Hours & Cons. & Inv. & Output \\
\hline \multirow{4}{*}{ Norm } & \multirow{2}{*}{ Unc. } & -54.75 & -37.48 & -90.61 & -45.99 & -50.02 & -35.73 & -49.42 & -38.46 \\
& \multirow{2}{*}{ Dis. } & $(17.88)$ & $(12.5)$ & $(28.21)$ & $(14.24)$ & & & & \\
& & -6.7 & -1.86 & -23.9 & -4.22 & -19.74 & -5.73 & -48.57 & -14.27 \\
& $(2.48)$ & $(2.02)$ & $(4.75)$ & $(2.17)$ & & & & \\
\hline \multirow{2}{*}{ Beta } & Unc. & -15.82 & -4.92 & -29.18 & -9.31 & -16.77 & -2.15 & -52.28 & -12.06 \\
& \multirow{2}{*}{ Dis. } & $(24.17)$ & $(15.1)$ & $(38.26)$ & $(18.57)$ & & & & \\
& & -5.82 & -1.63 & -21.6 & -3.84 & -4.42 & -1.60 & -9.26 & -3.12 \\
\hline \hline
\end{tabular}

Table B.2: Data and model predicted effects of uncertainty and disagreement on aggregate variables for normal and beta distributional fits. $\theta$ is calibrated to fit an evenly weighted sum of the effect of uncertainty and disagreement on labor and consumption. Aggregate variables represented as percent deviations from steady state. Productivity, uncertainty, and disagreement represented in percent. Newey-West standard errors from the regression shown in parenthesis.

(ECB) which also conducts a Survey of Professional Forecasters. Started in 1999, the ECB SPF is conducted quarterly. Survey respondents are experts employed by financial or nonfinancial institutions such as economic research institutions. Similar to the US SPF, the ECB SPF asks for point and density forecasts for the annual-average over annual-average growth rate of real GDP. The respondents provide the probability that the growth rate falls into a number of bins. To be consistent with the US SPF data, I analyze forecasts for current calendar year. The properties of the data are

- The number of respondents per year ranges from 34 to 44

- The width of bins is fixed at .5\%.

- The number of bins varies from a minimum of 10 to a maximum of 22. From 1991Q1 to 2000Q1 the bins ranged from 0\% to 4\%. From 2000Q2 to 2000Q4 the bins ranged from 0\% to 5\%. From 2001Q1 to 2008Q3 the bins ranged from 0\% to 4\%. From 2008Q4 to 2009Q1 the bins ranged from $-1 \%$ to 4\%. From 2009Q2 to 2009Q4 the bins ranged from $-6 \%$ to $4 \%$. From $2010 \mathrm{Q} 1$ to the present the bins ranged from $-1 \%$ to $4 \%$. 

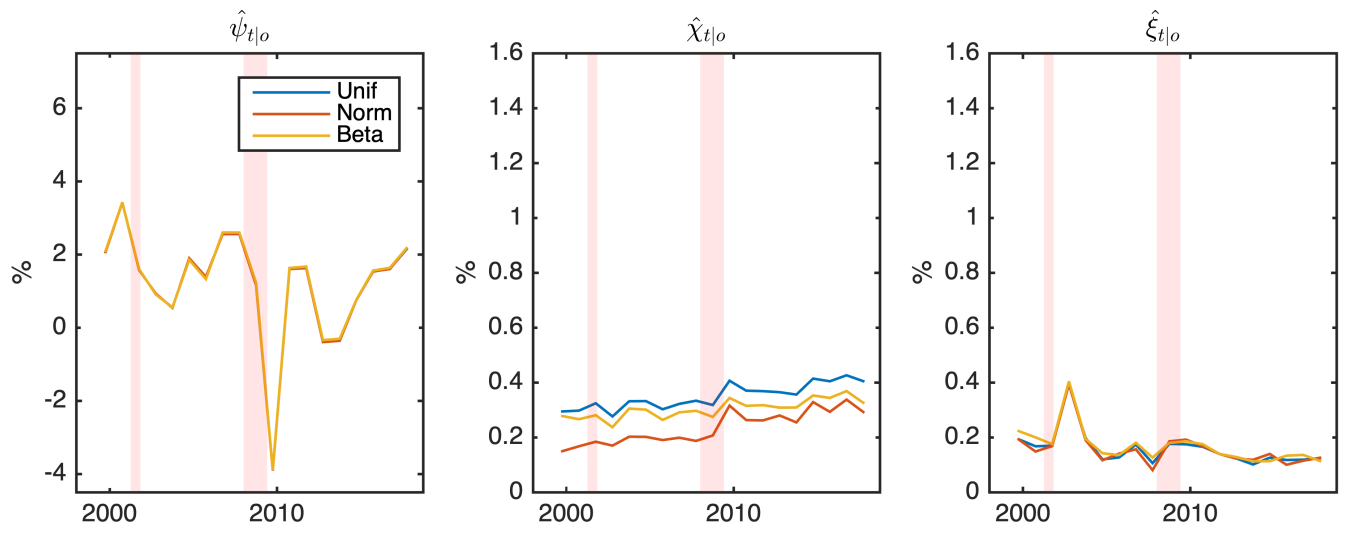

Figure B.3: Plot comparing estimates of $\hat{\psi}_{t \mid o}, \hat{\chi}_{t \mid o}$, and $\hat{\xi}_{t \mid o}$ using a uniform, normal, and generalized beta distribution with $o=4$ for ECB data. Blue shows the uniform fit results, red shows the normal fit results, and yellow shows the beta fit results. Red highlights show NBER recession periods. All values are annualized and in percent.

Following the same methodology applied to the US SPF data, I compute estimates for $\hat{\psi}_{t \mid o}$, $\hat{\chi}_{t \mid o}$, and $\hat{\xi}_{t \mid o}$ using the ECB SPF data and compare the fits for a uniform, normal, and beta distributional assumption in Figure B.3. Note that similar to the results from the US SPF data, the fits line up very well for $\hat{\psi}_{t \mid o}$. There is a downward bias in the normal distribution fit for $\hat{\chi}_{t \mid o}$, while the volatility of the beta fit is higher in the plot for $\hat{\xi}_{t \mid o}$. I find this to be suggestive that the binning structure does not have large effects in my results.

\section{B.3 Robustness of Properties of Uncertainty and Disagreement}

In order to verify the qualitative time series properties of uncertainty, I use the MSC (Michigan Survey of Consumers). Started in the 1950s, The MSC is a telephone survey conducted monthly by the University of Michigan of at least 500 households across the continental US. Wtih response rates varying between $60 \%-70 \%$, the survey consists of 50 core questions which ask about household's own economic situation as well as their forecasts of the future state of the economy. I focus on the question which asks households to forecast business conditions for the country as a whole in the next 12 months. The responses are 

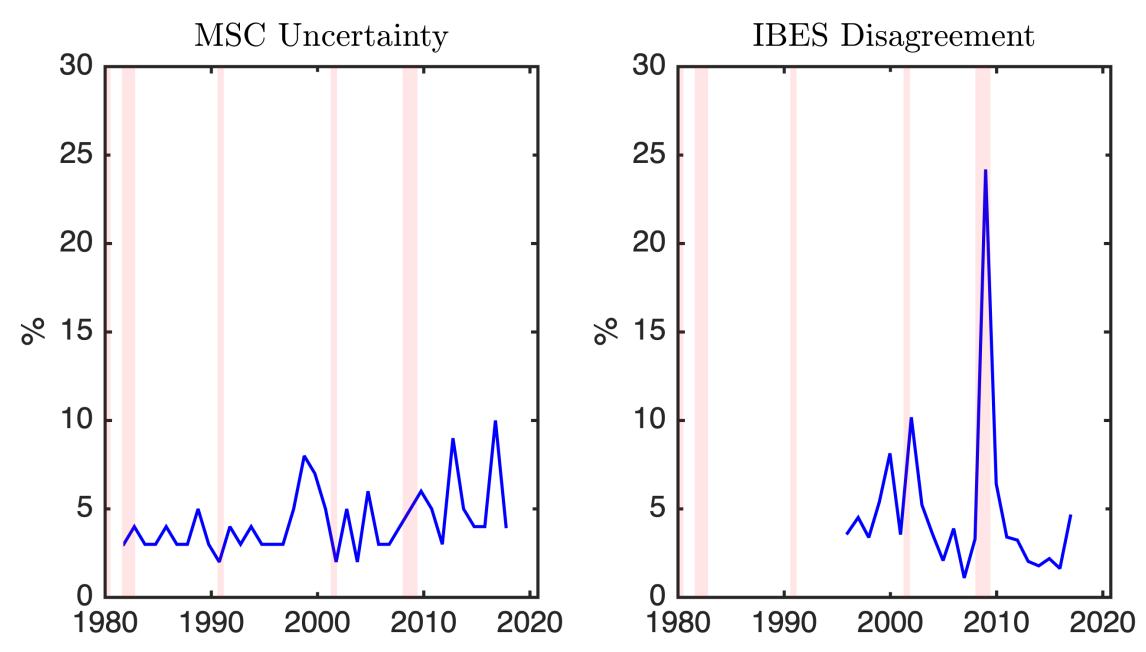

Figure B.4: Plot of estimates of uncertainty from the MSC and disagreement from IBES. Red highlights show NBER recession periods. All values are annualized and in percent.

classified by the survey into five categories: "good times", "uncertain", "bad times", "don't know", and "not applicable." I take the percentage of respondents that answered "uncertain" as a proxy for the dispersion within each agent's individual agents forecast for economic conditions. Thus I find it is a proxy for uncertainty. To match the range of SPF data, I use quarterly data in the range 1981-2017.

I verify the time-series properties of disagreement using the Thompson Reuters Institutional Brokers Estimate System (IBES). Started in 1976, the IBES database collects future earnings estimates for US companies made by institutional brokers. The database initially started with annual earnings estimates then was expanded to the quarterly earnings. Each forecaster is asked to provide a point forecast of the earnings on various US companies that are publicly traded. I focus on forecasts of operating earnings per share of the S\&P500 index. To be consistent with SPF data, I use 1 year ahead forecasts of the annual earnings estimates. Due to data availability, the time-series data from IBES is at an annual frequency and only spans 1995-2017 with the number of respondents varying from 3 to 19. I take the standard deviation of the point forecast scaled by the mean forecast across the forecasters 


\begin{tabular}{r|rrrr}
\hline \hline & Hours Worked & Consumption & Investment & Output \\
\hline Uncertainty & $-82.09^{* *}$ & $-53.83^{* *}$ & $-140.51^{* *}$ & $-67.09^{* *}$ \\
& $(22.56)$ & $(16.78)$ & $(39.5)$ & $(19.59)$ \\
Disagreement & $19.48^{*}$ & $17.04^{* *}$ & 28.27 & $17.71^{*}$ \\
& $(10.59)$ & $(6.55)$ & $(19.58)$ & $(8.53)$ \\
TFP & 0.07 & $0.3^{* *}$ & $0.57^{* *}$ & $0.29^{* *}$ \\
& $(0.1)$ & $(0.09)$ & $(0.14)$ & $(0.1)$ \\
\hline$R^{2}$ & 0.35 & 0.56 & 0.44 & 0.49 \\
$\mathrm{~N}$ & 25 & 25 & 25 & 25 \\
\hline \hline
\end{tabular}

Table B.3: Effects of uncertainty and disagreement on US aggregate variables excluding year 2009. Uniform distributional assumption used. Aggregate variables represented as percent deviations from steady state. Productivity, uncertainty, and disagreement represented in percent. Newey-West robust standard errors shown.

as a proxy for disagreement.

In Figure B.4, I plot the proxies for uncertainty and disagreement from the MSC and IBES databases. Note that similar to the results from the SPF, uncertainty from the MSC does not vary much or change in recession times. Disagreement from IBES is highly procyclical with the disagreement across IBES forecasters spiking during the recession periods.

\section{B.4 Role of Recent Financial Crisis}

In my model, aggregate variation is driven almost entirely by TFP shocks. Looking at the properties of disagreement, I observe a large spike during the 2008 financial crisis. Given there were many other factors besides productivity which played a role in the financial crisis, I run the reduced form regression of uncertainty and disagreement on aggregate variables excluding the spike in year 2009 using the specification in Equation (6.1). I use the uniform distributional assumption in extracting uncertainty and disagreement. I show the results in Table B.3.

I find that excluding the year 2009, my regression results exhibit notable changes. First, I find that an increase in disagreement causes a positive shock to aggregate variables in contrast to my results including the year 2009. In addition, the magnitude of the effects of 
uncertainty on aggregate variables is amplified. The evidence suggests my interpretation of the effect of disagreement is subject to the fact that I attribute the large disagreement in 2009 as affecting macrovariables entirely through the posterior about productivity. Given the limited availability of data, while these results do not rule out my model mechanism, I acknowledge it does not account for demand shocks or financial effects which occurred during this time period. I leave the inclusion of these effects as future work. 


\section{APPENDIX C}

\section{APPENDIX: LINKING REAL GDP GROWTH IN SURVEY DATA TO PRODUCTIVITY IN THE MODEL}

\section{C.1 Forecasting Real GDP Growth with Aggregate Labor Change}

Let $r_{t}$ denote the real GDP growth rate in my model. I find as before

$$
r_{t}=\frac{\exp \left(s_{t}\right) K_{t}^{\alpha} N_{t}^{1-\alpha}}{\exp \left(s_{t-1}\right) K_{t-1}^{\alpha} N_{t-1}^{1-\alpha}}-1
$$

I set the economy to be in steady state at $t-1$, but now allow the forecasts of real GDP to potentially depend only on aggregate labor. The growth rate is then approximated by

$$
\begin{aligned}
r_{1, t} & \approx s_{1, t}+\frac{1-\alpha}{\bar{N}} N_{1, t} \\
& =s_{1, t}+\frac{1-\alpha}{\bar{N}}\left[n_{k} K_{1, t}+n_{K} K_{1, t}+n_{z} \zeta_{1, t}+n_{a} a_{1, t}+n_{b} b_{1, t}+n_{s^{-}} s_{1, t-1}+n_{\zeta^{-}} \zeta_{1, t-1}\right] \\
& =s_{1, t}+\frac{1-\alpha}{\bar{N}}\left[n_{z} \zeta_{1, t}+n_{a} a_{1, t}+n_{b} b_{1, t}\right]
\end{aligned}
$$

where in the second equality, I have plugged in the first order approximation for aggregate labor. $\bar{N}$ represents the steady state aggregate labor and $\left\{n_{k}, n_{K}, \ldots\right\}$ represent the coefficient from the first order solution to the optimal labor choice on the state variables. Since I assume the system is in steady state in $t-1$, most of the state dependence drops out.

Looking at the expression for the growth rate of real GDP, I find that the expected growth rate of real GDP depends on $s_{t}, \zeta_{t}, a_{t}, b_{t}$. In Stage 1 of time $t$ since $a_{t}, b_{t}$ are common and known to all agents, agents' forecasts for $r_{t}$ will thus vary across agents in their posterior distributions for $s_{t}, \zeta_{t}$. Plugging in the posterior distribution for $s_{t}, \zeta_{t}$, I find that taking the 
first and second moments over $r_{t}$ under the undistorted measure

$$
\begin{aligned}
\mathbb{E}_{i, t}\left[r_{t}\right] & \approx\left(\frac{\sigma_{s}^{2}-a_{t}^{2}}{\sigma_{s}^{2}}+\frac{1-\alpha}{\bar{N}} n_{z} \frac{\sigma_{s}^{2}-a_{t}^{2}-b_{t}^{2}}{\sigma_{s}^{2}-a_{t}^{2}}\right) z_{i, t}+(\cdots) \\
\operatorname{Var}_{i, t}\left[r_{t}\right] & \approx\left[\begin{array}{ll}
1 & \frac{1-\alpha}{\bar{N}} n_{z}
\end{array}\right] \Sigma_{t}^{s, \zeta}\left[\begin{array}{c}
1 \\
\frac{1-\alpha}{N} n_{z}
\end{array}\right]
\end{aligned}
$$

where the ellipsis includes the contributions from the dependence on $a_{t}, b_{t}$.

Consistent with my definitions for uncertainty and disagreement which took moments of the distribution of forecasts for productivity, I compute corresponding moments for the distribution of forecasts for the expected growth rate of real GDP implied by my model. Let $\chi_{t}, \xi_{t}$ denote the standard deviation of each individual's expectation of $r_{t}$, and the standard deviation across agents of each agent's mean expectation for $r_{t}$ respectively. I find

$$
\begin{aligned}
\chi_{t} & \equiv\left(\left[1 \frac{1-\alpha}{\bar{N}} n_{z}\right] \Sigma_{t}^{s, \zeta}\left[\begin{array}{c}
1 \\
\frac{1-\alpha}{\bar{N}} n_{z}
\end{array}\right]\right)^{1 / 2} \\
\xi_{t} & \equiv\left(\frac{\sigma_{s}^{2}-a_{t}^{2}}{\sigma_{s}^{2}}+\frac{1-\alpha}{\bar{N}} n_{z} \frac{\sigma_{s}^{2}-a_{t}^{2}-b_{t}^{2}}{\sigma_{s}^{2}-a_{t}^{2}}\right) \frac{b_{t} \sigma_{s}^{2}}{\sigma_{s}^{2}-a_{t}^{2}}
\end{aligned}
$$

I note that my definitions of $\chi_{t}, \xi_{t}$ are independent of whether expectations are taken under the undistorted or distorted measure. From Equation (3.20), I find that the distortion adds a mean shift to the distribution of $s_{t}, \zeta_{t}$. However, this mean shift is a function of the covariance matrix $\Sigma_{t}^{s, \zeta}$ which is a function of $a_{t}, b_{t}$ and does not depend on $z_{i, t}$. As a result, the expression for $\xi_{t}$ would be unaffected since $a_{t}, b_{t}$ are common to all agents. Also, the distortion does not affect the variance of $s_{t}, \zeta_{t}$, and therefore, the expression for $\chi_{t}$ is also unaffected whether the undistorted or distorted densities are used.

My definitions of $\chi_{t}, \xi_{t}$ vary only from my definitions of uncertainty and disagreement through the endogenous term $n_{z}$. This reflects the fact that in forecasting the real GDP 

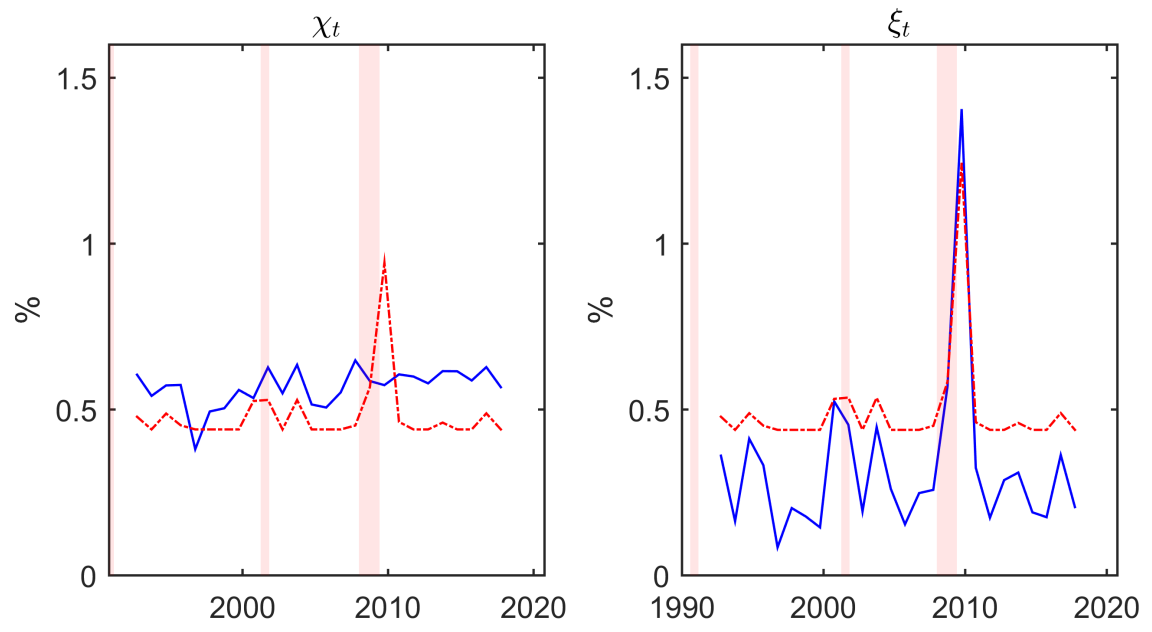

Figure C.1: Plot of fitted $\chi_{t}, \xi_{t}$ minimizing the objective in Equation (C.9) and the estimators $\hat{\chi}_{t \mid o}, \hat{\xi}_{t \mid o}$. I plot the estimators in blue, and the fitted values in dotted red. Red highlights indicate NBER recession periods. All variables annualized and in percent.

growth rate, each agent must take into account the effect that aggregate labor adjusts to beliefs about productivity. Setting $n_{z}=0$, I find then that $\chi_{t}, \xi_{t}$ coincide exactly with uncertainty and disagreement.

To fit $a_{t}, b_{t}$ dynamics incorporating the effects of aggregate labor, I set my estimators $\hat{\chi}_{t \mid s}, \hat{\xi}_{t \mid s}$ equal to my defined $\chi_{t}, \xi_{t}$ using Equations (C.7) and (C.8). For each time period $t$, I numerically search for the values of $a_{t}, b_{t}$ which minimize the objective

$$
\min _{a_{t}, b_{t}}\left(\chi_{t}\left(a_{t}, b_{t}\right)-\hat{\chi}_{t \mid o}\right)^{2}+\left(\xi_{t}\left(a_{t}, b_{t}\right)-\hat{\xi}_{t \mid o}\right)^{2}
$$

I plot the $\chi_{t}, \xi_{t}$ fitting this objective along with my estimates from the SPF data in Figure C.1.

Given the dependence on aggregate labor and structure I put on the signals in my model, I am unable to fit $a_{t}, b_{t}$ to match my estimators. I find the fit to be especially bad in 2009 , as I cannot match the spike in $\hat{\xi}_{t \mid o}$. Thus, to calibrate my model, I use the simplified estimator given in Equation (4.2) which allows me to fit the dynamics of $a_{t}, b_{t}$. 


\begin{tabular}{r|rr}
\hline \hline & $1960-2017$ & $1992-2017$ \\
\hline Output & $1.07^{* *}$ & $0.7^{* *}$ \\
& $(0.27)$ & $(0.31)$ \\
\hline$R^{2}$ & 0.22 & 0.18 \\
$\mathrm{~N}$ & 58 & 26 \\
\hline \hline
\end{tabular}

Table C.1: Contemporary regression of TFP on real Output. All variables represented as percent deviation from steady state. Results for the full sample 1960-2017 and the sample consistent with SPF data 1992-2017 shown. Spherical standard errors shown.

\section{C.2 Comparing Real GDP Growth and Productivity}

To provide additional support for my use of uncertainty and disagreement measured from forecasts of real GDP growth rate predictions as applied to productivity $s_{t}$ in my model. I run tests comparing the growth rate to TFP.

In Table C.1, I run a contemporary regression of TFP on real output using US aggregate data. To transform the series to be stationary, I remove a linear trend from each variables and represent each as a percent deviation from steady state. I find this regression has a coefficient significant at the $1 \%$ level and $R^{2}=.22$ using annual data over the sample of 1960-2017. Restricting the regression to the sample of 1992-2017 consistent with the SPF data on real GDP growth, I find the coefficient is significant at the $5 \%$ level and $R^{2}=.18$ . The result suggests that real output capture a significant fraction of relevant variation of TFP.

I then run a regression of TFP on the average predicted real GDP growth rate from the SPF data. To transform the variables, I measure TFP annually as a percent change from the previous year. I use the moment $\hat{\psi}_{\left.t\right|_{o}} \equiv \frac{1}{J_{t}} \sum_{j=1}^{J_{t}} \mu_{t \mid o}^{j}$ with the uniform distributional assumption to measure the average predicted growth rate. I find this regression has a nonsignificant coefficient and an $R^{2}$ of near zero. This result suggests the mean real GDP growth rate from survey responses is a poor predictor of TFP. However, my extracted uncertainty and disagreement do not rely on the accuracy of the average prediction but properties of the dispersion both within and across forecasters. I note that the approximation of using real 
GDP growth rate in the survey and applying it to TFP in the model is crude, but I find there is some support which links the two variables together. 